\title{
NONHARMONIC ANALYSIS OF BOUNDARY VALUE PROBLEMS WITHOUT WZ CONDITION
}

\author{
MICHAEL RUZHANSKY AND NIYAZ TOKMAGAMBETOV
}

\begin{abstract}
In this work we continue our research on nonharmonic analysis of boundary value problems as initiated in [RT16]. There, we assumed that the eigenfunctions of the model operator on which the construction is based do not have zeros. In this paper we have weakened this condition extending the applicability of the developed pseudo-differential analysis. Also, we do not assume that the underlying set $\Omega$ is bounded.
\end{abstract}

\section{INTRODUCTION}

In [RT16] the authors developed pseudo-differential calculus in terms of the 'model' densely defined operator L. The main examples are operators in $\Omega \subset \mathbb{R}^{n}$ equipped with (arbitrary) boundary conditions on $\partial \Omega$ for which the global Fourier analysis in terms of its eigenfunctions was introduced. Such a 'model' operator L does not have to be self-adjoint, so the construction is based on biorthogonal systems rather than on an orthonormal basis (to take into account the non-self-adjointness). Also, the operator L does not have to be elliptic. The 'model' operator L was considered as a differential operator of order $m$ with smooth coefficients on an open bounded set $\Omega \subset \mathbb{R}^{n}$ equipped with some boundary conditions which one can denote as (BC). In [RT16] one worked with discrete sets of eigenvalues and eigenfunctions indexed by a countable set, and one developed elements of the symbolic calculus assuming that the system of eigenfunctions is the without zeros in $\Omega$ (so called WZ-system). We refer to [RT16] for examples and an extensive list of references in this subject.

In this paper we will drop some conditions of the 'model' operator L. Let us consider the case when $\mathrm{L}$ is an arbitrary operator in $\Omega \subseteq \mathbb{R}^{n}$ with the discrete spectrum and the system of eigenfunctions which is a Riesz basis in $L^{2}(\Omega)$.

Denote the corresponding countable index set by $\mathcal{I}$. However, in different problems it may be more convenient to make different choices for this set, e.g. $\mathcal{I}=\mathbb{N}$ or $\mathbb{Z}$ or $\mathbb{Z}^{k}$, etc. In order to allow different applications we will be denoting it by $\mathcal{I}$, and without loss of generality we will assume that

$$
\mathcal{I} \text { is a subset of } \mathbb{Z}^{K} \text { for some } K \geq 1 \text {. }
$$

Date: August 7, 2018.

1991 Mathematics Subject Classification. Primary 58J40; Secondary 35S05, 35S30, 42B05.

Key words and phrases. Pseudo-differential operators, boundary value problems, Fourier series, non-local boundary condition, nonharmonic analysis.

The first author was supported in parts by the EPSRC grant EP/K039407/1 and by the Leverhulme Grant RPG-2014-02. The second author was supported in parts by the MESRK grant 0773/GF4. No new data was collected or generated during the course of this research. 
For simplicity, one can think of $\mathcal{I}=\mathbb{Z}$ or $\mathcal{I}=\mathbb{N} \cup\{0\}$ throughout this paper. Thus, in this paper we will be always working in the following setting:

Assumption 1.1. Let $\Omega \subseteq \mathbb{R}^{n}, n \geq 1$, be an open set. Assume that $\mathrm{L}$ is a densely defined operator with a discrete spectrum $\left\{\lambda_{\xi} \in \mathbb{C}: \xi \in \mathcal{I}\right\}$ on $L^{2}(\Omega)$, and the system of corresponding eigenfunctions $\left\{u_{\xi}: \xi \in \mathcal{I}\right\}$ is a Riesz basis in $L^{2}(\Omega)$ (i.e. for every $f \in L^{2}(\Omega)$ there exists a unique series $\sum_{\xi \in \mathcal{I}} a_{\xi} u_{\xi}(x)$ that converges to $f$ in $L^{2}(\Omega)$ ), where $\mathcal{I}$ is a countable set as in (1.1), and we order the eigenvalues with the occurring multiplicities in the ascending order:

$$
\left|\lambda_{j}\right| \leq\left|\lambda_{k}\right| \quad \text { for }|j| \leq|k| \text {. }
$$

We denote by $u_{\xi}$ the eigenfunction of L corresponding to the eigenvalue $\lambda_{\xi}$ for each $\xi \in \mathcal{I}$, so that

$$
\mathrm{L} u_{\xi}=\lambda_{\xi} u_{\xi} \quad \text { in } \Omega, \quad \text { for all } \xi \in \mathcal{I} .
$$

The conjugate spectral problem is

$$
\mathrm{L}^{*} v_{\xi}=\bar{\lambda}_{\xi} v_{\xi} \quad \text { in } \Omega \quad \text { for all } \xi \in \mathcal{I} \text {. }
$$

Let $\left\|u_{\xi}\right\|_{L^{2}}=1$ and $\left\|v_{\xi}\right\|_{L^{2}}=1$ for all $\xi \in \mathcal{I}$. Here, we can take biorthogonal systems $\left\{u_{\xi}\right\}_{\xi \in \mathcal{I}}$ and $\left\{v_{\xi}\right\}_{\xi \in \mathcal{I}}$, i.e.

$$
\left(u_{\xi}, v_{\eta}\right)_{L^{2}}=0 \text { for } \xi \neq \eta \text {, and }\left(u_{\xi}, v_{\eta}\right)_{L^{2}}=1 \text { for } \xi=\eta
$$

where

$$
(f, g)_{L^{2}}:=\int_{\Omega} f(x) \overline{g(x)} d x
$$

is the usual inner product of the Hilbert space $L^{2}(\Omega)$. From N.K. Bari's work [Bar51] it follows that the system $\left\{u_{\xi}: \xi \in \mathcal{I}\right\}$ is a basis in $L^{2}(\Omega)$ if and only if the system $\left\{v_{\xi}: \xi \in \mathcal{I}\right\}$ is a basis in $L^{2}(\Omega)$. Therefore, by Bari [Bar51], the system $\left\{v_{\xi}: \xi \in \mathcal{I}\right\}$ is also a basis in $L^{2}(\Omega)$. Also, Assumption 1.1 will imply that the spaces $C_{\mathrm{L}}^{\infty}(\bar{\Omega})$ and $C_{\mathrm{L}^{*}}^{\infty}(\bar{\Omega})$ of test functions introduced in Subsection 2 are dense in $L^{2}(\Omega)$.

Define the weight

$$
\langle\xi\rangle:=\left(1+\left|\lambda_{\xi}\right|^{2}\right)^{\frac{1}{2 m}},
$$

which will be instrumental in measuring the growth/decay of Fourier coefficients and of symbols. Here $m>0$ is an arbitrary number that we fix throughout the paper. For simplicity we can take $m=1$. However, if $\mathrm{L}$ is, for example, a differential operator, it is convenient to take $m$ to be equal to its order.

To give the interpretation for $\langle\xi\rangle$ in terms of the operator analysis, we can define the operator $\mathrm{L}^{\circ}$ by setting its values on the basis $u_{\xi}$ by

$$
\mathrm{L}^{\circ} u_{\xi}:=\overline{\lambda_{\xi}} u_{\xi}, \quad \text { for all } \xi \in \mathcal{I} .
$$

If $\mathrm{L}$ is self-adjoint, we have $\mathrm{L}^{\circ}=\mathrm{L}^{*}=\mathrm{L}$. Consequently, we can informally think of $\langle\xi\rangle$ as of the eigenvalues of the positive (first order) operator $\left(\mathrm{I}+\mathrm{L}^{\circ} \mathrm{L}\right)^{\frac{1}{2 m}}$.

With a similar definition for $\left(\mathrm{L}^{*}\right)^{\circ}$, we can observe that $\left(\mathrm{L}^{*}\right)^{\circ}=\left(\mathrm{L}^{\circ}\right)^{*}$.

Simplest examples of non-periodic boundary conditions were considered in [KTT15] and [KT14] in the case of $\Omega=[0,1]$ being the segment. This extends to the nonperiodic case the periodic analysis developed in [RT07, RT09, RT10a, RT10b] on the 
torus which can be viewed as analysis on $\Omega=[0,1]$ with periodic boundary conditions. We refer to [RT16] for further examples.

\section{Preliminary}

In this section we collect some results on L-distributions, L-Fourier transform, Plancherel formula and Sobolev spaces $\mathcal{H}_{\mathrm{L}}^{s}(\Omega)$, and we omit the proofs because they are a straightforward extension of those in [RT16].

2.1. Global distributions generated by the boundary value problem. In this subsection we describe the spaces of distributions generated by $\mathrm{L}$ and by its adjoint $\mathrm{L}^{*}$ and the related global Fourier analysis. The more far-reaching aim of this analysis is to establish a version of the Schwartz kernel theorem for the appearing spaces of distributions. We first define the space $C_{\mathrm{L}}^{\infty}(\bar{\Omega})$ of test functions.

Definition 2.1. The space $C_{\mathrm{L}}^{\infty}(\bar{\Omega}):=\operatorname{Dom}\left(\mathrm{L}^{\infty}\right)$ is called the space of test functions for L. Here we define

$$
\operatorname{Dom}\left(\mathrm{L}^{\infty}\right):=\bigcap_{k=1}^{\infty} \operatorname{Dom}\left(\mathrm{L}^{k}\right),
$$

where $\operatorname{Dom}\left(\mathrm{L}^{k}\right)$ is the domain of the operator $\mathrm{L}^{k}$, in turn defined as

$$
\operatorname{Dom}\left(\mathrm{L}^{k}\right):=\left\{f \in L^{2}(\Omega): \mathrm{L}^{j} f \in \operatorname{Dom}(\mathrm{L}), j=0,1,2, \ldots, k-1\right\} .
$$

The Fréchet topology of $C_{\mathrm{L}}^{\infty}(\bar{\Omega})$ is given by the family of norms

$$
\|\varphi\|_{C_{\mathrm{L}}^{k}}:=\max _{j \leq k}\left\|\mathrm{~L}^{j} \varphi\right\|_{L^{2}(\Omega)}, \quad k \in \mathbb{N}_{0}, \varphi \in C_{\mathrm{L}}^{\infty}(\bar{\Omega}) .
$$

Analogously to the L-case, we introduce the space $C_{\mathrm{L}^{*}}^{\infty}(\bar{\Omega})$ corresponding to the adjoint operator $\mathrm{L}_{\Omega}^{*}$ by

$$
C_{\mathrm{L}^{*}}^{\infty}(\bar{\Omega}):=\operatorname{Dom}\left(\left(\mathrm{L}^{*}\right)^{\infty}\right)=\bigcap_{k=1}^{\infty} \operatorname{Dom}\left(\left(\mathrm{L}^{*}\right)^{k}\right),
$$

where $\operatorname{Dom}\left(\left(\mathrm{L}^{*}\right)^{k}\right)$ is the domain of the operator $\left(\mathrm{L}^{*}\right)^{k}$,

$$
\operatorname{Dom}\left(\left(\mathrm{L}^{*}\right)^{k}\right):=\left\{f \in L^{2}(\Omega):\left(\mathrm{L}^{*}\right)^{j} f \in \operatorname{Dom}\left(\mathrm{L}^{*}\right), \quad j=0, \ldots, k-1\right\},
$$

which satisfy the adjoint boundary conditions corresponding to the operator $\mathrm{L}_{\Omega}^{*}$. The Fréchet topology of $C_{\mathrm{L}^{*}}^{\infty}(\bar{\Omega})$ is given by the family of norms

$$
\|\psi\|_{C_{\mathrm{L}^{*}}^{k}}:=\max _{j \leq k}\left\|\left(\mathrm{~L}^{*}\right)^{j} \psi\right\|_{L^{2}(\Omega)}, \quad k \in \mathbb{N}_{0}, \psi \in C_{\mathrm{L}^{*}}^{\infty}(\bar{\Omega}) .
$$

Since we have $u_{\xi} \in C_{\mathrm{L}}^{\infty}(\bar{\Omega})$ and $v_{\xi} \in C_{\mathrm{L}^{*}}^{\infty}(\bar{\Omega})$ for all $\xi \in \mathcal{I}$, we observe that Assumption 1.1 implies that the spaces $C_{\mathrm{L}}^{\infty}(\bar{\Omega})$ and $C_{\mathrm{L}^{*}}^{\infty}(\bar{\Omega})$ are dense in $L^{2}(\Omega)$.

We note that if $\mathrm{L}$ is self-adjoint, i.e. if $\mathrm{L}^{*}=\mathrm{L}$ with the equality of domains, then $C_{\mathrm{L}^{*}}^{\infty}(\bar{\Omega})=C_{\mathrm{L}}^{\infty}(\bar{\Omega})$.

In general, for functions $f \in C_{\mathrm{L}}^{\infty}(\bar{\Omega})$ and $g \in C_{\mathrm{L}^{*}}^{\infty}(\bar{\Omega})$, the $L^{2}$-duality makes sense in view of the formula

$$
(\mathrm{L} f, g)_{L^{2}(\Omega)}=\left(f, \mathrm{~L}^{*} g\right)_{L^{2}(\Omega)}
$$

Therefore, in view of the formula (2.3), it makes sense to define the distributions $\mathcal{D}_{\mathrm{L}}^{\prime}(\Omega)$ as the space which is dual to $C_{\mathrm{L}^{*}}^{\infty}(\bar{\Omega})$. 
Definition 2.2. The space

$$
\mathcal{D}_{\mathrm{L}}^{\prime}(\Omega):=\mathcal{L}\left(C_{\mathrm{L}^{*}}^{\infty}(\bar{\Omega}), \mathbb{C}\right)
$$

of linear continuous functionals on $C_{\mathrm{L}^{*}}^{\infty}(\bar{\Omega})$ is called the space of L-distributions. We can understand the continuity here either in terms of the topology (2.2) or in terms of sequences, see Proposition 2.3. For $w \in \mathcal{D}_{\mathrm{L}}^{\prime}(\Omega)$ and $\varphi \in C_{\mathrm{L}^{*}}^{\infty}(\bar{\Omega})$, we shall write

$$
w(\varphi)=\langle w, \varphi\rangle \text {. }
$$

For any $\psi \in C_{\mathrm{L}}^{\infty}(\bar{\Omega})$,

$$
C_{\mathrm{L}^{*}}^{\infty}(\bar{\Omega}) \ni \varphi \mapsto \int_{\Omega} \psi(x) \varphi(x) d x
$$

is an L-distribution, which gives an embedding $\psi \in C_{\mathrm{L}}^{\infty}(\bar{\Omega}) \hookrightarrow \mathcal{D}_{\mathrm{L}}^{\prime}(\Omega)$. We note that in the distributional notation formula (2.3) becomes

$$
\langle\mathrm{L} \psi, \varphi\rangle=\left\langle\psi, \overline{\mathrm{L}^{*} \bar{\varphi}}\right\rangle .
$$

With the topology on $C_{\mathrm{L}}^{\infty}(\bar{\Omega})$ defined by $(2.1)$, the space

$$
\mathcal{D}_{\mathrm{L}^{*}}^{\prime}(\Omega):=\mathcal{L}\left(C_{\mathrm{L}}^{\infty}(\bar{\Omega}), \mathbb{C}\right)
$$

of linear continuous functionals on $C_{\mathrm{L}}^{\infty}(\bar{\Omega})$ is called the space of $\mathrm{L}^{*}$-distributions.

Proposition 2.3. A linear functional $w$ on $C_{\mathrm{L}^{*}}^{\infty}(\bar{\Omega})$ belongs to $\mathcal{D}_{\mathrm{L}}^{\prime}(\Omega)$ if and only if there exists a constant $c>0$ and a number $k \in \mathbb{N}_{0}$ with the property

$$
|w(\varphi)| \leq c\|\varphi\|_{C_{\mathrm{L}^{*}}^{k}} \quad \text { for all } \varphi \in C_{\mathrm{L}^{*}}^{\infty}(\bar{\Omega}) .
$$

The space $\mathcal{D}_{\mathrm{L}}^{\prime}(\Omega)$ has many similarities with the usual spaces of distributions. For example, suppose that for a linear continuous operator $D: C_{\mathrm{L}}^{\infty}(\bar{\Omega}) \rightarrow C_{\mathrm{L}}^{\infty}(\bar{\Omega})$ its adjoint $D^{*}$ preserves the domain of $\mathrm{L}^{*}$ and is continuous on the space $C_{\mathrm{L}^{*}}^{\infty}(\bar{\Omega})$, i.e. that the operator $D^{*}: C_{\mathrm{L}^{*}}^{\infty}(\bar{\Omega}) \rightarrow C_{\mathrm{L}^{*}}^{\infty}(\bar{\Omega})$ is continuous. Then we can extend $D$ to $\mathcal{D}_{\mathrm{L}}^{\prime}(\Omega)$ by

$$
\langle D w, \varphi\rangle:=\left\langle w, \overline{D^{*} \bar{\varphi}}\right\rangle \quad\left(w \in \mathcal{D}_{\mathrm{L}}^{\prime}(\Omega), \varphi \in C_{\mathrm{L}^{*}}^{\infty}(\bar{\Omega})\right) .
$$

This extends (2.4) from L to other operators. The convergence in the linear space $\mathcal{D}_{\mathrm{L}}^{\prime}(\Omega)$ is the usual weak convergence with respect to the space $C_{\mathrm{L}^{*}}^{\infty}(\bar{\Omega})$. The following principle of uniform boundedness is based on the Banach-Steinhaus Theorem applied to the Fréchet space $C_{\mathrm{L}^{*}}^{\infty}(\bar{\Omega})$.

Lemma 2.4. Let $\left\{w_{j}\right\}_{j \in \mathbb{N}}$ be a sequence in $\mathcal{D}_{\mathrm{L}}^{\prime}(\Omega)$ with the property that for every $\varphi \in C_{\mathrm{L}^{*}}^{\infty}(\bar{\Omega})$, the sequence $\left\{w_{j}(\varphi)\right\}_{j \in \mathbb{N}}$ in $\mathbb{C}$ is bounded. Then there exist constants $c>0$ and $k \in \mathbb{N}_{0}$ such that

$$
\left|w_{j}(\varphi)\right| \leq c\|\varphi\|_{C_{\mathrm{L}^{*}}^{k}} \quad \text { for all } j \in \mathbb{N}, \varphi \in C_{\mathrm{L}^{*}}^{\infty}(\bar{\Omega}) .
$$

The lemma above leads to the following property of completeness of the space of L-distributions.

Theorem 2.5. Let $\left\{w_{j}\right\}_{j \in \mathbb{N}}$ be a sequence in $\mathcal{D}_{\mathrm{L}}^{\prime}(\Omega)$ with the property that for every $\varphi \in C_{\mathrm{L}^{*}}^{\infty}(\bar{\Omega})$ the sequence $\left\{w_{j}(\varphi)\right\}_{j \in \mathbb{N}}$ converges in $\mathbb{C}$ as $j \rightarrow \infty$. Denote the limit by $w(\varphi)$. 
(i) Then $w: \varphi \mapsto w(\varphi)$ defines an L-distribution on $\Omega$. Furthermore,

$$
\lim _{j \rightarrow \infty} w_{j}=w \quad \text { in } \quad \mathcal{D}_{\mathrm{L}}^{\prime}(\Omega)
$$

(ii) If $\varphi_{j} \rightarrow \varphi$ in $\in C_{\mathrm{L}^{*}}^{\infty}(\bar{\Omega})$, then

$$
\lim _{j \rightarrow \infty} w_{j}\left(\varphi_{j}\right)=w(\varphi) \quad \text { in } \quad \mathbb{C} .
$$

Similarly to the previous case, we have analogues of Proposition 2.3 and Theorem 2.5 for $\mathrm{L}^{*}$-distributions.

2.2. L-Fourier transform. In this subsection we define the L-Fourier transform generated by our operator $\mathrm{L}$ and its main properties. The main difference between the self-adjoint and non-self-adjoint problems $\mathrm{L}$ is that in the latter case we have to make sure that we use the right functions from the available biorthogonal families of $u_{\xi}$ and $v_{\xi}$. We start by defining the spaces that we will obtain on the Fourier transform side.

Let $\mathcal{S}(\mathcal{I})$ denote the space of rapidly decaying functions $\varphi: \mathcal{I} \rightarrow \mathbb{C}$. That is, $\varphi \in \mathcal{S}(\mathcal{I})$ if for any $M<\infty$ there exists a constant $C_{\varphi, M}$ such that

$$
|\varphi(\xi)| \leq C_{\varphi, M}\langle\xi\rangle^{-M}
$$

holds for all $\xi \in \mathcal{I}$. Here $\langle\xi\rangle$ is already adapted to our case since it is defined by (1.6).

The topology on $\mathcal{S}(\mathcal{I})$ is given by the seminorms $p_{k}$, where $k \in \mathbb{N}_{0}$ and

$$
p_{k}(\varphi):=\sup _{\xi \in \mathcal{I}}\langle\xi\rangle^{k}|\varphi(\xi)| \text {. }
$$

Continuous linear functionals on $\mathcal{S}(\mathcal{I})$ are of the form

$$
\varphi \mapsto\langle u, \varphi\rangle:=\sum_{\xi \in \mathcal{I}} u(\xi) \varphi(\xi)
$$

where functions $u: \mathcal{I} \rightarrow \mathbb{C}$ grow at most polynomially at infinity, i.e. there exist constants $M<\infty$ and $C_{u, M}$ such that

$$
|u(\xi)| \leq C_{u, M}\langle\xi\rangle^{M}
$$

holds for all $\xi \in \mathcal{I}$. Such distributions $u: \mathcal{I} \rightarrow \mathbb{C}$ form the space of distributions which we denote by $\mathcal{S}^{\prime}(\mathcal{I})$. We now define the L-Fourier transform on $C_{\mathrm{L}}^{\infty}(\bar{\Omega})$.

Definition 2.6. We define the L-Fourier transform

$$
\left(\mathcal{F}_{\mathrm{L}} f\right)(\xi)=(f \mapsto \widehat{f}): C_{\mathrm{L}}^{\infty}(\bar{\Omega}) \rightarrow \mathcal{S}(\mathcal{I})
$$

by

$$
\widehat{f}(\xi):=\left(\mathcal{F}_{\mathrm{L}} f\right)(\xi)=\int_{\Omega} f(x) \overline{v_{\xi}(x)} d x .
$$

Analogously, we define the L*-Fourier transform

$$
\left(\mathcal{F}_{\mathrm{L}^{*}} f\right)(\xi)=\left(f \mapsto \widehat{f}_{*}\right): C_{\mathrm{L}^{*}}^{\infty}(\bar{\Omega}) \rightarrow \mathcal{S}(\mathcal{I})
$$

by

$$
\widehat{f}_{*}(\xi):=\left(\mathcal{F}_{\mathrm{L}^{*}} f\right)(\xi)=\int_{\Omega} f(x) \overline{u_{\xi}(x)} d x
$$


The expressions (2.7) and (2.8) are well-defined by the Cauchy-Schwarz inequality, for example,

$$
|\widehat{f}(\xi)|=\left|\int_{\Omega} f(x) \overline{v_{\xi}(x)} d x\right| \leq\|f\|_{L^{2}}\left\|v_{\xi}\right\|_{L^{2}}=\|f\|_{L^{2}}<\infty .
$$

Moreover, we have

Proposition 2.7. The L-Fourier transform $\mathcal{F}_{\mathrm{L}}$ is a bijective homeomorphism from $C_{\mathrm{L}}^{\infty}(\bar{\Omega})$ to $\mathcal{S}(\mathcal{I})$. Its inverse

$$
\mathcal{F}_{\mathrm{L}}^{-1}: \mathcal{S}(\mathcal{I}) \rightarrow C_{\mathrm{L}}^{\infty}(\bar{\Omega})
$$

is given by

$$
\left(\mathcal{F}_{\mathrm{L}}^{-1} h\right)(x)=\sum_{\xi \in \mathcal{I}} h(\xi) u_{\xi}(x), \quad h \in \mathcal{S}(\mathcal{I}),
$$

so that the Fourier inversion formula becomes

$$
f(x)=\sum_{\xi \in \mathcal{I}} \widehat{f}(\xi) u_{\xi}(x) \quad \text { for all } f \in C_{\mathrm{L}}^{\infty}(\bar{\Omega}) .
$$

Similarly, $\mathcal{F}_{\mathrm{L}^{*}}: C_{\mathrm{L}^{*}}^{\infty}(\bar{\Omega}) \rightarrow \mathcal{S}(\mathcal{I})$ is a bijective homeomorphism and its inverse

$$
\mathcal{F}_{\mathrm{L}^{*}}^{-1}: \mathcal{S}(\mathcal{I}) \rightarrow C_{\mathrm{L}^{*}}^{\infty}(\bar{\Omega})
$$

is given by

$$
\left(\mathcal{F}_{\mathrm{L}^{*}}^{-1} h\right)(x):=\sum_{\xi \in \mathcal{I}} h(\xi) v_{\xi}(x), \quad h \in \mathcal{S}(\mathcal{I})
$$

so that the conjugate Fourier inversion formula becomes

$$
f(x)=\sum_{\xi \in \mathcal{I}} \widehat{f}_{*}(\xi) v_{\xi}(x) \quad \text { for all } f \in C_{\mathrm{L}^{*}}^{\infty}(\bar{\Omega}) .
$$

By dualising the inverse L-Fourier transform $\mathcal{F}_{\mathrm{L}}^{-1}: \mathcal{S}(\mathcal{I}) \rightarrow C_{\mathrm{L}}^{\infty}(\bar{\Omega})$, the L-Fourier transform extends uniquely to the mapping

$$
\mathcal{F}_{\mathrm{L}}: \mathcal{D}_{\mathrm{L}}^{\prime}(\Omega) \rightarrow \mathcal{S}^{\prime}(\mathcal{I})
$$

by the formula

$$
\left\langle\mathcal{F}_{\mathrm{L}} w, \varphi\right\rangle:=\left\langle w, \overline{\mathcal{F}_{\mathrm{L}^{*}}^{-1} \bar{\varphi}}\right\rangle, \quad \text { with } w \in \mathcal{D}_{\mathrm{L}}^{\prime}(\Omega), \varphi \in \mathcal{S}(\mathcal{I}) .
$$

It can be readily seen that if $w \in \mathcal{D}_{\mathrm{L}}^{\prime}(\Omega)$ then $\widehat{w} \in \mathcal{S}^{\prime}(\mathcal{I})$. The reason for taking complex conjugates in (2.14) is that, if $w \in C_{\mathrm{L}}^{\infty}(\bar{\Omega})$, we have the equality

$$
\begin{aligned}
\langle\widehat{w}, \varphi\rangle=\sum_{\xi \in \mathcal{I}} \widehat{w}(\xi) \varphi(\xi)=\sum_{\xi \in \mathcal{I}}\left(\int_{\Omega} w(x) \overline{v_{\xi}(x)} d x\right) \varphi(\xi) \\
=\int_{\Omega} w(x) \overline{\left(\sum_{\xi \in \mathcal{I}} \overline{\varphi(\xi)} v_{\xi}(x)\right)} d x=\int_{\Omega} w(x) \overline{\left(\mathcal{F}_{\mathrm{L}^{*}}^{-1} \bar{\varphi}\right)} d x=\left\langle w, \overline{\mathcal{F}_{\mathrm{L}^{*}}^{-1} \bar{\varphi}}\right\rangle .
\end{aligned}
$$

Analogously, we have the mapping

$$
\mathcal{F}_{\mathrm{L}^{*}}: \mathcal{D}_{\mathrm{L}^{*}}^{\prime}(\Omega) \rightarrow \mathcal{S}^{\prime}(\mathcal{I})
$$


defined by the formula

$$
\left\langle\mathcal{F}_{\mathrm{L}^{*}} w, \varphi\right\rangle:=\left\langle w, \overline{\mathcal{F}_{\mathrm{L}}^{-1} \bar{\varphi}}\right\rangle, \quad \text { with } w \in \mathcal{D}_{\mathrm{L}^{*}}^{\prime}(\Omega), \varphi \in \mathcal{S}(\mathcal{I}) .
$$

It can be also seen that if $w \in \mathcal{D}_{\mathrm{L}^{*}}^{\prime}(\Omega)$ then $\widehat{w} \in \mathcal{S}^{\prime}(\mathcal{I})$.

We note that since systems of $u_{\xi}$ and of $v_{\xi}$ are Riesz bases, we can also compare $L^{2}$-norms of functions with sums of squares of Fourier coefficients. The following statement follows from the work of Bari [Bar51, Theorem 9]:

Lemma 2.8. There exist constants $k, K, m, M>0$ such that for every $f \in L^{2}(\Omega)$ we have

$$
m^{2}\|f\|_{L^{2}}^{2} \leq \sum_{\xi \in \mathcal{I}}|\widehat{f}(\xi)|^{2} \leq M^{2}\|f\|_{L^{2}}^{2}
$$

and

$$
k^{2}\|f\|_{L^{2}}^{2} \leq \sum_{\xi \in \mathcal{I}}\left|\widehat{f}_{*}(\xi)\right|^{2} \leq K^{2}\|f\|_{L^{2}}^{2} .
$$

However, we note that the Plancherel identity can be also achieved in suitably defined $l^{2}$-spaces of Fourier coefficients, see Proposition 2.9.

2.3. Plancherel formula, Sobolev spaces $\mathcal{H}_{\mathrm{L}}^{s}(\Omega)$, and their Fourier images. In this subsection we discuss Sobolev spaces adapted to L and their images under the L-Fourier transform. We start with the $L^{2}$-setting, where we can recall inequalities between $L^{2}$-norms of functions and sums of squares of their Fourier coefficients, see Lemma 2.8. However, below we show that we actually have the Plancherel identity in a suitably defined space $l_{\mathrm{L}}^{2}$ and its conjugate $l_{\mathrm{L}^{*}}^{2}$.

Let us denote by

$$
l_{\mathrm{L}}^{2}=l^{2}(\mathrm{~L})
$$

the linear space of complex-valued functions $a$ on $\mathcal{I}$ such that $\mathcal{F}_{\mathrm{L}}^{-1} a \in L^{2}(\Omega)$, i.e. if there exists $f \in L^{2}(\Omega)$ such that $\mathcal{F}_{\mathrm{L}} f=a$. Then the space of sequences $l_{\mathrm{L}}^{2}$ is a Hilbert space with the inner product

$$
(a, b)_{l_{\mathrm{L}}^{2}}:=\sum_{\xi \in \mathcal{I}} a(\xi) \overline{\left(\mathcal{F}_{\mathrm{L}^{*}} \circ \mathcal{F}_{\mathrm{L}}^{-1} b\right)(\xi)}
$$

for arbitrary $a, b \in l_{\mathrm{L}}^{2}$. The reason for this choice of the definition is the following formal calculation:

$$
\begin{aligned}
(a, b)_{l_{\mathrm{L}}^{2}} & =\sum_{\xi \in \mathcal{I}} a(\xi) \overline{\left(\mathcal{F}_{\mathrm{L}^{*}} \circ \mathcal{F}_{\mathrm{L}}^{-1} b\right)(\xi)} \\
& =\sum_{\xi \in \mathcal{I}} a(\xi) \int_{\Omega} \overline{\left(\mathcal{F}_{\mathrm{L}}^{-1} b\right)(x)} u_{\xi}(x) d x \\
& =\int_{\Omega}\left[\sum_{\xi \in \mathcal{I}} a(\xi) u_{\xi}(x)\right] \overline{\left(\mathcal{F}_{\mathrm{L}}^{-1} b\right)(x)} d x \\
& =\int_{\Omega}\left(\mathcal{F}_{\mathrm{L}}^{-1} a\right)(x) \overline{\left(\mathcal{F}_{\mathrm{L}}^{-1} b\right)(x)} d x \\
& =\left(\mathcal{F}_{\mathrm{L}}^{-1} a, \mathcal{F}_{\mathrm{L}}^{-1} b\right)_{L^{2}}
\end{aligned}
$$


which implies the Hilbert space properties of the space of sequences $l_{\mathrm{L}}^{2}$. The norm of $l_{\mathrm{L}}^{2}$ is then given by the formula

$$
\|a\|_{l_{\mathrm{L}}^{2}}=\left(\sum_{\xi \in \mathcal{I}} a(\xi) \overline{\left(\mathcal{F}_{\mathrm{L}^{*}} \circ \mathcal{F}_{\mathrm{L}}^{-1} a\right)(\xi)}\right)^{1 / 2}, \quad \text { for all } a \in l_{\mathrm{L}}^{2} .
$$

We note that individual terms in this sum may be complex-valued but the whole sum is real and nonnegative due to formula (2.17).

Analogously, we introduce the Hilbert space

$$
l_{\mathrm{L}^{*}}^{2}=l^{2}\left(\mathrm{~L}^{*}\right)
$$

as the space of functions $a$ on $\mathcal{I}$ such that $\mathcal{F}_{\mathrm{L}^{*}}^{-1} a \in L^{2}(\Omega)$, with the inner product

$$
(a, b)_{l_{\mathrm{L}^{*}}^{2}}:=\sum_{\xi \in \mathcal{I}} a(\xi) \overline{\left(\mathcal{F}_{\mathrm{L}} \circ \mathcal{F}_{\mathrm{L}^{*}}^{-1} b\right)(\xi)}
$$

for arbitrary $a, b \in l_{\mathrm{L}^{*}}^{2}$. The norm of $l_{\mathrm{L}^{*}}^{2}$ is given by the formula

$$
\|a\|_{l_{\mathrm{L}^{*}}^{2}}=\left(\sum_{\xi \in \mathcal{I}} a(\xi) \overline{\left(\mathcal{F}_{\mathrm{L}} \circ \mathcal{F}_{\mathrm{L}^{*}}^{-1} a\right)(\xi)}\right)^{1 / 2}
$$

for all $a \in l_{\mathrm{L}^{*}}^{2}$. The spaces of sequences $l_{\mathrm{L}}^{2}$ and $l_{\mathrm{L}^{*}}^{2}$ are thus generated by biorthogonal systems $\left\{u_{\xi}\right\}_{\xi \in \mathcal{I}}$ and $\left\{v_{\xi}\right\}_{\xi \in \mathcal{I}}$. The reason for their definition in the above forms becomes clear again in view of the following Plancherel identity:

Proposition 2.9. (Plancherel's identity) If $f, g \in L^{2}(\Omega)$ then $\widehat{f}, \widehat{g} \in l_{\mathrm{L}}^{2}, \widehat{f}_{*}, \widehat{g}_{*} \in l_{\mathrm{L}^{*}}^{2}$, and the inner products (2.16), (2.19) take the form

$$
(\widehat{f}, \widehat{g})_{l_{\mathrm{L}}^{2}}=\sum_{\xi \in \mathcal{I}} \widehat{f}(\xi) \overline{\widehat{g}_{*}(\xi)}
$$

and

$$
\left(\widehat{f}_{*}, \widehat{g}_{*}\right)_{l_{\mathrm{L}^{*}}^{2}}=\sum_{\xi \in \mathcal{I}} \widehat{f}_{*}(\xi) \overline{\widehat{g}(\xi)}
$$

In particular, we have

$$
\overline{(\widehat{f}, \widehat{g})_{l_{\mathrm{L}}^{2}}}=\left(\widehat{g}_{*}, \widehat{f}_{*}\right)_{l_{\mathrm{L}^{*}}^{2}} \text {. }
$$

The Parseval identity takes the form

$$
(f, g)_{L^{2}}=(\widehat{f}, \widehat{g})_{l_{\mathrm{L}}^{2}}=\sum_{\xi \in \mathcal{I}} \widehat{f}(\xi) \overline{\widehat{g}_{*}(\xi)} .
$$

Furthermore, for any $f \in L^{2}(\Omega)$, we have $\widehat{f} \in l_{\mathrm{L}}^{2}, \widehat{f}_{*} \in l_{\mathrm{L}^{*}}^{2}$, and

$$
\|f\|_{L^{2}}=\|\widehat{f}\|_{l_{\mathrm{L}}^{2}}=\left\|\widehat{f}_{*}\right\|_{l_{\mathrm{L}^{*}}^{2}} .
$$

Now we introduce Sobolev spaces generated by the operator L:

Definition 2.10 (Sobolev spaces $\mathcal{H}_{\mathrm{L}}^{s}(\Omega)$ ). For $f \in \mathcal{D}_{\mathrm{L}}^{\prime}(\Omega) \cap \mathcal{D}_{\mathrm{L}^{*}}^{\prime}(\Omega)$ and $s \in \mathbb{R}$, we say that

$$
f \in \mathcal{H}_{\mathrm{L}}^{s}(\Omega) \text { if and only if }\langle\xi\rangle^{s} \widehat{f}(\xi) \in l_{\mathrm{L}}^{2}
$$


We define the norm on $\mathcal{H}_{\mathrm{L}}^{s}(\Omega)$ by

$$
\|f\|_{\mathcal{H}_{\mathrm{L}}^{s}(\Omega)}:=\left(\sum_{\xi \in \mathcal{I}}\langle\xi\rangle^{2 s} \widehat{f}(\xi) \overline{\widehat{f}_{*}(\xi)}\right)^{1 / 2} .
$$

The Sobolev space $\mathcal{H}_{\mathrm{L}}^{s}(\Omega)$ is then the space of L-distributions $f$ for which we have $\|f\|_{\mathcal{H}_{\mathrm{L}}^{s}(\Omega)}<\infty$. Similarly, we can define the space $\mathcal{H}_{\mathrm{L}^{*}}^{s}(\Omega)$ by the condition

$$
\|f\|_{\mathcal{H}_{\mathrm{L}^{*}}^{s}(\Omega)}:=\left(\sum_{\xi \in \mathcal{I}}\langle\xi\rangle^{2 s} \widehat{f}_{*}(\xi) \overline{\widehat{f}(\xi)}\right)^{1 / 2}<\infty .
$$

We note that the expressions in (2.22) and (2.23) are well-defined since the sum

$$
\sum_{\xi \in \mathcal{I}}\langle\xi\rangle^{2 s} \widehat{f}(\xi) \overline{\widehat{f}_{*}(\xi)}=\left(\langle\xi\rangle^{s} \widehat{f}(\xi),\langle\xi\rangle^{s} \widehat{f}(\xi)\right)_{l_{\mathrm{L}}^{2}} \geq 0
$$

is real and non-negative. Consequently, since we can write the sum in (2.23) as the complex conjugate of that in (2.22), and with both being real, we see that the spaces $\mathcal{H}_{\mathrm{L}}^{s}(\Omega)$ and $\mathcal{H}_{\mathrm{L}^{*}}^{s}(\Omega)$ coincide as sets. Moreover, we have

Proposition 2.11. For every $s \in \mathbb{R}$, the Sobolev space $\mathcal{H}_{\mathrm{L}}^{s}(\Omega)$ is a Hilbert space with the inner product

$$
(f, g)_{\mathcal{H}_{\mathrm{L}}^{s}(\Omega)}:=\sum_{\xi \in \mathcal{I}}\langle\xi\rangle^{2 s} \widehat{f}(\xi) \overline{\widehat{g}_{*}(\xi)}
$$

Similarly, the Sobolev space $\mathcal{H}_{\mathrm{L}^{*}}^{s}(\Omega)$ is a Hilbert space with the inner product

$$
(f, g)_{\mathcal{H}_{\mathrm{L}^{*}}^{s}(\Omega)}:=\sum_{\xi \in \mathcal{I}}\langle\xi\rangle^{2 s} \widehat{f}_{*}(\xi) \overline{\widehat{g}(\xi)} .
$$

For every $s \in \mathbb{R}$, the Sobolev spaces $\mathcal{H}^{s}(\Omega), \mathcal{H}_{\mathrm{L}}^{s}(\Omega)$, and $\mathcal{H}_{\mathrm{L}^{*}}^{s}(\Omega)$ are isometrically isomorphic.

2.4. Spaces $l^{p}(\mathrm{~L})$ and $l^{p}\left(\mathrm{~L}^{*}\right)$. In this subsection we describe the $p$-Lebesgue versions of the spaces of Fourier coefficients. These spaces can be considered as the extension of the usual $l^{p}$ spaces on the discrete set $\mathcal{I}$ adapted to the fact that we are dealing with biorthogonal systems.

Definition 2.12. Thus, we introduce the spaces $l_{\mathrm{L}}^{p}=l^{p}(\mathrm{~L})$ as the spaces of all $a \in \mathcal{S}^{\prime}(\mathcal{I})$ such that

$$
\|a\|_{l^{p}(\mathrm{~L})}:=\left(\sum_{\xi \in \mathcal{I}}|a(\xi)|^{p}\left\|u_{\xi}\right\|_{L^{\infty}(\Omega)}^{2-p}\right)^{1 / p}<\infty, \quad \text { for } 1 \leq p \leq 2,
$$

and

$$
\|a\|_{l^{p}(\mathrm{~L})}:=\left(\sum_{\xi \in \mathcal{I}}|a(\xi)|^{p}\left\|v_{\xi}\right\|_{L^{\infty}(\Omega)}^{2-p}\right)^{1 / p}<\infty, \quad \text { for } 2 \leq p<\infty,
$$

and, for $p=\infty$,

$$
\|a\|_{l^{\infty}(\mathrm{L})}:=\sup _{\xi \in \mathcal{I}}\left(|a(\xi)| \cdot\left\|v_{\xi}\right\|_{L^{\infty}(\Omega)}^{-1}\right)<\infty .
$$


Remark 2.13. We note that in the case of $p=2$, we have already defined the space $l^{2}(\mathrm{~L})$ by the norm (2.18). There is no problem with this since the norms (2.24)-(2.25) with $p=2$ are equivalent to that in (2.18). Indeed, by Lemma 2.8 the first one gives a homeomorphism between $l^{p}(\mathrm{~L})$ with $p=2$ just defined and $L^{2}(\Omega)$ while the space $l^{2}(\mathrm{~L})$ defined by $(2.18)$ is isometrically isomorphic to $L^{2}(\Omega)$ by the Plancherel identity in Proposition 2.9. Therefore, both norms lead to the same space which we denote by $l^{2}(\mathrm{~L})$. The norms (2.24)-(2.25) with $p=2$ and the one in (2.18) are equivalent, but there are advantages in using both of them. Thus, the norms (2.24)(2.25) allow us to view $l^{2}(\mathrm{~L})$ as a member of the scale of spaces $l^{p}(\mathrm{~L})$ for $1 \leq p \leq \infty$ with subsequent functional analytic properties, while the norm (2.18) is the one for which the Plancherel identity (2.21) holds.

Analogously, we also introduce spaces $l_{\mathrm{L}^{*}}^{p}=l^{p}\left(\mathrm{~L}^{*}\right)$ as the spaces of all $b \in \mathcal{S}^{\prime}(\mathcal{I})$ such that the following norms are finite:

$$
\begin{aligned}
\|b\|_{l^{p}\left(\mathrm{~L}^{*}\right)}= & \left(\sum_{\xi \in \mathcal{I}}|b(\xi)|^{p}\left\|v_{\xi}\right\|_{L^{\infty}(\Omega)}^{2-p}\right)^{1 / p}, \quad \text { for } 1 \leq p \leq 2, \\
\|b\|_{l^{p}\left(\mathrm{~L}^{*}\right)}= & \left(\sum_{\xi \in \mathcal{I}}|b(\xi)|^{p}\left\|u_{\xi}\right\|_{L^{\infty}(\Omega)}^{2-p}\right)^{1 / p}, \quad \text { for } \quad 2 \leq p<\infty, \\
& \|b\|_{l^{\infty}\left(\mathrm{L}^{*}\right)}=\sup _{\xi \in \mathcal{I}}\left(|b(\xi)| \cdot\left\|u_{\xi}\right\|_{L^{\infty}(\Omega)}^{-1}\right) .
\end{aligned}
$$

Before we discuss several basic properties of the spaces $l^{p}(\mathrm{~L})$, we recall a useful fact on the interpolation of weighted spaces from Bergh and Löfström [BL76, Theorem 5.5.1]:

Theorem 2.14 (Interpolation of weighted spaces). Let us write $d \mu_{0}(x)=\omega_{0}(x) d \mu(x)$, $d \mu_{1}(x)=\omega_{1}(x) d \mu(x)$, and write $L^{p}(\omega)=L^{p}(\omega d \mu)$ for the weight $\omega$. Suppose that $0<p_{0}, p_{1}<\infty$. Then

$$
\left(L^{p_{0}}\left(\omega_{0}\right), L^{p_{1}}\left(\omega_{1}\right)\right)_{\theta, p}=L^{p}(\omega)
$$

where $0<\theta<1, \frac{1}{p}=\frac{1-\theta}{p_{0}}+\frac{\theta}{p_{1}}$, and $\omega=\omega_{0}^{\frac{p(1-\theta)}{p_{0}}} \omega_{1}^{\frac{p \theta}{p_{1}}}$.

From this it is easy to check that we obtain:

Corollary 2.15 (Interpolation of $l^{p}(\mathrm{~L})$ and $l^{p}\left(\mathrm{~L}^{*}\right)$ spaces). For $1 \leq p \leq 2$, we have

$$
\begin{gathered}
\left(l^{1}(\mathrm{~L}), l^{2}(\mathrm{~L})\right)_{\theta, p}=l^{p}(\mathrm{~L}), \\
\left(l^{1}\left(\mathrm{~L}^{*}\right), l^{2}\left(\mathrm{~L}^{*}\right)\right)_{\theta, p}=l^{p}\left(\mathrm{~L}^{*}\right),
\end{gathered}
$$

where $0<\theta<1$ and $p=\frac{2}{2-\theta}$.

Remark 2.16. The reason that the interpolation above is restricted to $1 \leq p \leq 2$ is that the definition of $l^{p}$-spaces changes when we pass $p=2$, in the sense that we use different families of biorthogonal systems $u_{\xi}$ and $v_{\xi}$ for $p<2$ and for $p>2$. We note that if $\mathrm{L}=\mathrm{L}^{*}$ is self-adjoint, so that we can take $u_{\xi}=v_{\xi}$ for all $\xi \in \mathcal{I}$, then the scales $l^{p}(\mathrm{~L})$ and $l^{p}\left(\mathrm{~L}^{*}\right)$ coincide and satisfy interpolation properties for all $1 \leq p<\infty$.

Using these interpolation properties we can establish further properties of the Fourier transform and its inverse: 
Theorem 2.17 (Hausdorff-Young inequality). Let $1 \leq p \leq 2$ and $\frac{1}{p}+\frac{1}{p^{\prime}}=1$. There is a constant $C_{p} \geq 1$ such that for all $f \in L^{p}(\Omega)$ and $a \in l^{p}(\mathrm{~L})$ we have

$$
\|\widehat{f}\|_{l^{p^{\prime}(\mathrm{L})}} \leq C_{p}\|f\|_{L^{p}(\Omega)} \quad \text { and } \quad\left\|\mathcal{F}_{\mathrm{L}}^{-1} a\right\|_{L^{p^{\prime}(\Omega)}} \leq C_{p}\|a\|_{l^{p}(\mathrm{~L})} .
$$

Similarly, we also have

$$
\left\|\widehat{f}_{*}\right\|_{l^{p^{\prime}\left(\mathrm{L}^{*}\right)}} \leq C_{p}\|f\|_{L^{p}(\Omega)} \quad \text { and } \quad\left\|\mathcal{F}_{\mathrm{L}^{*}}^{-1} b\right\|_{L^{p^{\prime}}(\Omega)} \leq C_{p}\|b\|_{l^{p}\left(\mathrm{~L}^{*}\right)}
$$

for all $b \in l^{p}\left(\mathrm{~L}^{*}\right)$.

It follows from the proof that if $\mathrm{L}$ is self-adjoint, then the $l_{L}^{2}$-norms discussed in Remark 2.13 coincide, and so we can put $C_{p}=1$ in inequalities (2.26) and (2.27). If $\mathrm{L}$ is not self-adjoint, $C_{p}$ may in principle depend on $\mathrm{L}$ and its domain through constants from inequalities in Lemma 2.8.

We now turn to the duality between spaces $l^{p}(\mathrm{~L})$ and $l^{q}\left(\mathrm{~L}^{*}\right)$ :

Theorem 2.18 (Duality of $l^{p}(\mathrm{~L})$ and $l^{p^{\prime}}\left(\mathrm{L}^{*}\right)$ ). Let $1 \leq p<\infty$ and $\frac{1}{p}+\frac{1}{p^{\prime}}=1$. Then

$$
\left(l^{p}(\mathrm{~L})\right)^{\prime}=l^{p^{\prime}}\left(\mathrm{L}^{*}\right) \quad \text { and } \quad\left(l^{p}\left(\mathrm{~L}^{*}\right)\right)^{\prime}=l^{p^{\prime}}(\mathrm{L}) .
$$

2.5. Schwartz' kernel theorem. In our case the Schwartz kernel theorem is also valid and here we will briefly discuss it. So, from now on we will make the following:

Assumption 2.19. Assume that the number $s_{0} \in \mathbb{R}$ is such that we have

$$
\sum_{\xi \in \mathcal{I}}\langle\xi\rangle^{-s_{0}}<\infty
$$

Recalling the operator $\mathrm{L}^{\circ}$ in (1.7) the Assumption 2.19 is equivalent to assuming that the operator $\left(\mathrm{I}+\mathrm{L}^{\circ} \mathrm{L}\right)^{-\frac{s_{0}}{4 m}}$ is Hilbert-Schmidt on $L^{2}(\Omega)$. Indeed, recalling the definition of $\langle\xi\rangle$ in (1.6), namely that $\langle\xi\rangle$ are the eigenvalues of $\left(\mathrm{I}+\mathrm{L}^{\circ} \mathrm{L}\right)^{-\frac{s_{0}}{2 m}}$, the condition that the operator $\left(\mathrm{I}+\mathrm{L}^{\circ} \mathrm{L}\right)^{-\frac{s_{0}}{4 m}}$ is Hilbert-Schmidt is equivalent to the condition that

$$
\left\|\left(\mathrm{I}+\mathrm{L}^{\circ} \mathrm{L}\right)^{-\frac{s_{0}}{4 m}}\right\|_{\mathrm{HS}}^{2} \cong \sum_{\xi \in \mathcal{I}}\langle\xi\rangle^{-s_{0}}<\infty .
$$

If $\mathrm{L}$ is elliptic, we may expect that we can take any $s_{0}>n$ but this depends on the domain. The order $s_{0}$ will enter the regularity properties of the Schwartz kernels.

We will use the notations

$$
C_{\mathrm{L}}^{\infty}(\bar{\Omega} \times \bar{\Omega}):=C_{\mathrm{L}}^{\infty}(\bar{\Omega}) \bar{\otimes} C_{\mathrm{L}}^{\infty}(\bar{\Omega})
$$

and

$$
C_{\mathrm{L}^{*}}^{\infty}(\bar{\Omega} \times \bar{\Omega}):=C_{\mathrm{L}^{*}}^{\infty}(\bar{\Omega}) \bar{\otimes} C_{\mathrm{L}^{*}}^{\infty}(\bar{\Omega})
$$

with the Fréchet topologies given by the family of tensor norms

$$
\|\varphi \otimes \psi\|_{C_{\mathrm{L}}^{k}(\bar{\Omega} \times \bar{\Omega})}:=\max _{j+l \leq k}\left\|\mathrm{~L}^{j} \varphi\right\|_{L^{2}(\Omega)}\left\|\mathrm{L}^{l} \psi\right\|_{L^{2}(\Omega)}, \quad k \in \mathbb{N}_{0}, \varphi, \psi \in C_{\mathrm{L}}^{\infty}(\bar{\Omega})
$$

and

$$
\|\varphi \otimes \psi\|_{C_{\mathrm{L}^{*}}^{k}(\bar{\Omega} \times \bar{\Omega})}:=\max _{j+l \leq k}\left\|\left(\mathrm{~L}^{*}\right)^{j} \varphi\right\|_{L^{2}(\Omega)}\left\|\left(\mathrm{L}^{*}\right)^{l} \psi\right\|_{L^{2}(\Omega)}
$$


for all $k \in \mathbb{N}_{0}, \varphi, \psi \in C_{\mathrm{L}^{*}}^{\infty}(\bar{\Omega})$, respectively, and for the corresponding dual spaces we write

$$
\begin{aligned}
\mathcal{D}_{\mathrm{L}}^{\prime}(\Omega \times \Omega) & :=\left(C_{\mathrm{L}}^{\infty}(\bar{\Omega} \times \bar{\Omega})\right)^{\prime}, \\
\mathcal{D}_{\mathrm{L}^{*}}^{\prime}(\Omega \times \Omega) & :=\left(C_{\mathrm{L}^{*}}^{\infty}(\bar{\Omega} \times \bar{\Omega})\right)^{\prime} .
\end{aligned}
$$

For any linear continuous operator

$$
A: C_{\mathrm{L}}^{\infty}(\bar{\Omega}) \rightarrow \mathcal{D}_{\mathrm{L}}^{\prime}(\Omega)
$$

there exists a kernel $K_{A} \in \mathcal{D}_{\mathrm{L}}^{\prime}(\Omega \times \Omega)$ such that for all $f \in C_{\mathrm{L}}^{\infty}(\bar{\Omega})$, we can write, in the sense of distributions,

$$
A f(x)=\int_{\Omega} K_{A}(x, y) f(y) d y .
$$

As usual, $K_{A}$ is called the Schwartz kernel of $A$. For $f \in C_{\mathrm{L}}^{\infty}(\bar{\Omega})$, using the Fourier series formula

$$
f(y)=\sum_{\eta \in \mathcal{I}} \widehat{f}(\eta) u_{\eta}(y)
$$

we can also write

$$
A f(x)=\sum_{\eta \in \mathcal{I}} \widehat{f}(\eta) \int_{\Omega} K_{A}(x, y) u_{\eta}(y) d y .
$$

Also, for any linear continuous operator

$$
A: C_{\mathrm{L}^{*}}^{\infty}(\bar{\Omega}) \rightarrow \mathcal{D}_{\mathrm{L}^{*}}^{\prime}(\Omega)
$$

there exists a kernel $\widetilde{K}_{A} \in \mathcal{D}_{\mathrm{L}^{*}}^{\prime}(\Omega \times \Omega)$ such that for all $f \in C_{\mathrm{L}^{*}}^{\infty}(\bar{\Omega})$, we can write, in the sense of distributions,

$$
A f(x)=\int_{\Omega} \widetilde{K}_{A}(x, y) f(y) d y
$$

\section{L-ADMISSIBLE OPERATORS AND L-QUANTIZATION}

In this section we describe the L-quantization of the L-admissible operator induced by the operator L.

Definition 3.1. We say that the linear continuous operator

$$
A: C_{\mathrm{L}}^{\infty}(\bar{\Omega}) \rightarrow \mathcal{D}_{\mathrm{L}}^{\prime}(\Omega)
$$

belongs to the class of L-admissible operators if

$$
\sum_{\eta \in \mathcal{I}} u_{\eta}^{-1}(x) u_{\eta}(z) \int_{\Omega} K_{A}(x, y) u_{\eta}(y) d y
$$

is in $\mathcal{D}_{\mathrm{L}}^{\prime}(\Omega \times \Omega)$.

Remark 3.2. In the case when $\mathrm{L}$ is the Laplace operator with periodic boundary conditions on the torus $\mathbb{T}^{n}$ the class of L-admissible operators coincides with the class of all periodic pseudo-differential operators as in [RT10b].

So, from now on we will assume that operators $A: C_{\mathrm{L}}^{\infty}(\bar{\Omega}) \rightarrow \mathcal{D}_{\mathrm{L}}^{\prime}(\Omega)$ are from the class of L-admissible operators. 
Remark 3.3. Note, that the expression

$$
u_{\eta}^{-1}(x) \int_{\Omega} K_{A}(x, y) u_{\eta}(y) d y
$$

exists for any operator $A$ from the class of L-admissible operators. Moreover, it is in $\mathcal{D}_{\mathrm{L}}^{\prime}(\Omega) \otimes \mathcal{S}^{\prime}(\mathcal{I})$.

Indeed, since $\sum_{\eta \in \mathcal{I}} u_{\eta}^{-1}(x) u_{\eta}(z) \int_{\Omega} K_{A}(x, y) u_{\eta}(y) d y$ is in $\mathcal{D}_{\mathrm{L}}^{\prime}(\Omega \times \Omega)$, by taking Fourier transform in z, we get this statement. We now define the L-symbol of an L-admissible operator.

Definition 3.4 (L-Symbols of operators). The L-symbol of a linear continuous Ladmissible operator

$$
A: C_{\mathrm{L}}^{\infty}(\bar{\Omega}) \rightarrow \mathcal{D}_{\mathrm{L}}^{\prime}(\Omega)
$$

is defined by

$$
\sigma_{A}(x, \xi):=u_{\xi}^{-1}(x) \int_{\Omega} K_{A}(x, y) u_{\xi}(y) d y .
$$

This is well-defined as an element of $\mathcal{D}_{\mathrm{L}}^{\prime}(\Omega) \otimes \mathcal{S}^{\prime}(\mathcal{I})$ in view of Remark 3.3.

Indeed, we have

$$
A u_{\xi}=\int_{\Omega} K_{A}(x, y) u_{\xi}(y) d y
$$

and for $f \in C_{\mathrm{L}}^{\infty}(\bar{\Omega})$ from the expansion

$$
f(x)=\sum_{\xi \in \mathcal{I}} \widehat{f}(\xi) u_{\xi}(x)
$$

and by the operator $A: C_{\mathrm{L}}^{\infty}(\bar{\Omega}) \rightarrow \mathcal{D}_{\mathrm{L}}^{\prime}(\Omega)$ acting on $f$, we get

$$
A f(x)=\sum_{\xi \in \mathcal{I}} \widehat{f}(\xi) A u_{\xi}(x)=\sum_{\xi \in \mathcal{I}} \widehat{f}(\xi) \int_{\Omega} K_{A}(x, y) u_{\xi}(y) d y .
$$

Now, if we define

$$
u_{\xi}(x) \sigma_{A}(x, \xi):=\int_{\Omega} K_{A}(x, y) u_{\xi}(y) d y
$$

we have the implication

$$
K_{A}(x, y)=\sum_{\xi \in \mathcal{I}} u_{\xi}(x) \sigma_{A}(x, \xi) \overline{v_{\xi}(y)}
$$

Therefore we obtain the following representation of the operator $A$ by its symbol:

Theorem 3.5 (L-quantization). Let

$$
A: C_{\mathrm{L}}^{\infty}(\bar{\Omega}) \rightarrow \mathcal{D}_{\mathrm{L}}^{\prime}(\Omega)
$$

be a linear continuous $L$-admissible operator with $\mathrm{L}$-symbol $\sigma_{A} \in \mathcal{D}_{\mathrm{L}}^{\prime}(\Omega) \otimes \mathcal{S}^{\prime}(\mathcal{I})$. Then the $\mathrm{L}$-quantization

$$
A f(x)=\sum_{\xi \in \mathcal{I}} \widehat{f}(\xi) \sigma_{A}(x, \xi) u_{\xi}(x)
$$


is true for every $f \in C_{\mathrm{L}}^{\infty}(\bar{\Omega})$. The L-symbol $\sigma_{A}$ can be written as

$$
\sigma_{A}(x, \xi)=u_{\xi}^{-1}(x)\left(A u_{\xi}\right)(x) .
$$

Corollary 3.6. We have the following equivalent formulae for L-symbols:

$$
\begin{aligned}
\text { (i) } \sigma_{A}(x, \xi) & =u_{\xi}^{-1}(x)\left(A u_{\xi}\right)(x) \\
\text { (ii) } \sigma_{A}(x, \xi) & =u_{\xi}^{-1}(x) \int_{\Omega} K_{A}(x, y) u_{\xi}(y) d y
\end{aligned}
$$

Similarly, we can introduce an analogous notion of the $\mathrm{L}^{*}$-quantization.

Definition 3.7. We say that the continuous operator

$$
A: C_{\mathrm{L}^{*}}^{\infty}(\bar{\Omega}) \rightarrow \mathcal{D}_{\mathrm{L}^{*}}^{\prime}(\Omega)
$$

belongs to the class of $\mathrm{L}^{*}$-admissible operators if

$$
\sum_{\eta \in \mathcal{I}} v_{\eta}^{-1}(x) v_{\eta}(z) \int_{\Omega} \widetilde{K}_{A}(x, y) v_{\eta}(y) d y
$$

is in $\mathcal{D}_{\mathrm{L}^{*}}^{\prime}(\Omega \times \Omega)$.

Remark 3.8. Similarly to Remark 3.3, note that the expression

$$
v_{\xi}^{-1}(x) \int_{\Omega} \widetilde{K}_{A}(x, y) v_{\xi}(y) d y
$$

exists for any operator $A$ from the class of $\mathrm{L}^{*}$-admissible operators. Moreover, it is in $\mathcal{D}_{\mathrm{L}^{*}}^{\prime}(\Omega) \otimes \mathcal{S}^{\prime}(\mathcal{I})$.

We also can define the $\mathrm{L}^{*}$-symbol of an $\mathrm{L}^{*}$-admissible operator.

Definition 3.9. The $\mathrm{L}^{*}$-symbol of a linear continuous $\mathrm{L}^{*}$-admissible operator

$$
A: C_{\mathrm{L}^{*}}^{\infty}(\bar{\Omega}) \rightarrow \mathcal{D}_{\mathrm{L}^{*}}^{\prime}(\Omega)
$$

is defined by

$$
\tau_{A}(x, \xi):=v_{\xi}^{-1}(x) \int_{\Omega} \widetilde{K}_{A}(x, y) v_{\xi}(y) d y .
$$

Similarly to the case of L-symbols we have

$$
A v_{\xi}=\int_{\Omega} \widetilde{K}_{A}(x, y) v_{\xi}(y) d y,
$$

and for $f \in C_{\mathrm{L}^{*}}^{\infty}(\bar{\Omega})$ from the expantion

$$
f(x)=\sum_{\xi \in \mathcal{I}} \widehat{f}_{*}(\xi) v_{\xi}(x)
$$

and by the operator $A: C_{\mathrm{L}^{*}}^{\infty}(\bar{\Omega}) \rightarrow \mathcal{D}_{\mathrm{L}^{*}}^{\prime}(\Omega)$ acting on $f$, we get

$$
A f(x)=\sum_{\xi \in \mathcal{I}} \widehat{f}_{*}(\xi) A v_{\xi}(x)=\sum_{\xi \in \mathcal{I}} \widehat{f}_{*}(\xi) \int_{\Omega} \widetilde{K}_{A}(x, y) v_{\xi}(y) d y .
$$

Now, we have

$$
v_{\xi}(x) \tau_{A}(x, \xi):=\int_{\Omega} \widetilde{K}_{A}(x, y) v_{\xi}(y) d y
$$


hence also the implication

$$
\widetilde{K}_{A}(x, y)=\sum_{\xi \in \mathcal{I}} v_{\xi}(x) \tau_{A}(x, \xi) \overline{u_{\xi}(y)}
$$

We also record the resulting representation of the operator $A$ by its symbol:

Theorem 3.10. Let

$$
A: C_{\mathrm{L}^{*}}^{\infty}(\bar{\Omega}) \rightarrow \mathcal{D}_{\mathrm{L}^{*}}^{\prime}(\Omega)
$$

be a linear continuous $\mathrm{L}^{*}$-admissible operator with $\mathrm{L}^{*}$-symbol $\tau_{A} \in \mathcal{D}_{\mathrm{L}^{*}}^{\prime}(\Omega) \otimes \mathcal{S}^{\prime}(\mathcal{I})$. Then the $\mathrm{L}^{*}$-quantization is given by

$$
A f(x)=\sum_{\xi \in \mathcal{I}} \widehat{f}_{*}(\xi) \tau_{A}(x, \xi) v_{\xi}(x)
$$

for every $f \in C_{\mathrm{L}}^{\infty}(\bar{\Omega})$. The $\mathrm{L}^{*}$-symbol $\tau_{A}$ can be written as

$$
\tau_{A}(x, \xi)=v_{\xi}^{-1}(x)\left(A v_{\xi}\right)(x) .
$$

Corollary 3.11. We have the following equivalent formulae for $\mathrm{L}^{*}$-symbols:

$$
\begin{aligned}
\text { (i) } \tau_{A}(x, \xi) & =v_{\xi}^{-1}(x)\left(A v_{\xi}\right)(x) ; \\
\text { (ii) } \tau_{A}(x, \xi) & =v_{\xi}^{-1}(x) \int_{\Omega} \widetilde{K}_{A}(x, y) v_{\xi}(y) d y .
\end{aligned}
$$

We now briefly describe the notion of Fourier multipliers which is a natural name for operators with symbols independent of $x$. In [DRT15] the analysis of this paper is applied to investigate the spectral properties of such operators, so we can be brief here.

Definition 3.12. Let $A: C_{L}^{\infty}(\bar{\Omega}) \rightarrow C_{L}^{\infty}(\bar{\Omega})$ be a continuous linear operator. We will say that $A$ is an $L$-Fourier multiplier if it satisfies

$$
\mathcal{F}_{L}(A f)(\xi)=\sigma(\xi) \mathcal{F}_{L}(f)(\xi), f \in C_{L}^{\infty}(\bar{\Omega}),
$$

for some $\sigma: \mathcal{I} \rightarrow \mathbb{C}$. Analogously we define $L^{*}$-Fourier multipliers: Let $B: C_{L^{*}}^{\infty}(\bar{\Omega}) \rightarrow$ $C_{L^{*}}^{\infty}(\bar{\Omega})$ be a continuous linear operator. We will say that $B$ is an $L^{*}$-Fourier multiplier if it satisfies

$$
\mathcal{F}_{L^{*}}(B f)(\xi)=\tau(\xi) \mathcal{F}_{L^{*}}(f)(\xi), f \in C_{L^{*}}^{\infty}(\bar{\Omega}),
$$

for some $\tau: \mathcal{I} \rightarrow \mathbb{C}$.

As used in [DRT15], we have the following simple relation between the symbols of an operator and its adjoint.

Proposition 3.13. The operator $A$ is an L-Fourier multiplier by $\sigma(\xi)$ if and only if $A^{*}$ is an $L^{*}$-Fourier multiplier by $\overline{\sigma(\xi)}$.

\section{DifFERENCE OPERATORS}

In this section we discuss difference operators that will be instrumental in defining symbol classes for the symbolic calculus of operators.

Let $q_{j} \in C^{\infty}(\Omega \times \Omega), j=1, \ldots, l$, be a given family of smooth functions. We will call the collection of $q_{j}$ 's L-strongly admissible if the following properties hold: 
- The multiplication by $q_{j}(\cdot, \cdot)$ is a continuous linear mapping on $C_{\mathrm{L}^{*}}^{\infty}(\bar{\Omega} \times \bar{\Omega})$, for all $j=1, \ldots, l$;

- $q_{j}(x, x)=0$ and $\left.\nabla_{y} q_{j}(x, y)\right|_{y=x} \neq 0$ for all $j=1, \ldots, l$ and all $x \in \Omega$;

- $\left.\operatorname{rank}\left(\nabla_{y} q_{1}(x, y), \ldots, \nabla_{y} q_{l}(x, y)\right)\right|_{y=x}=n$ for all $x \in \Omega$;

- the diagonal in $\Omega \times \Omega$ is the only set when all of $q_{j}$ 's vanish:

$$
\bigcap_{j=1}^{l}\left\{(x, y) \in \Omega \times \Omega: q_{j}(x, y)=0\right\}=\{(x, x): x \in \Omega\} .
$$

We note that the first property above implies that for every $x \in \Omega$, the multiplication by $q_{j}(\cdot, \cdot)$ is also well-defined and extends to a continuous linear mapping on $\mathcal{D}_{\mathrm{L}}^{\prime}(\Omega \times \Omega)$. Also, the last property above contains the second one but we chose to still give it explicitly for the clarity of the exposition.

The collection of $q_{j}$ 's with the above properties generalises the notion of a strongly admissible collection of functions for difference operators introduced in [RTW14] in the context of compact Lie groups. We will use the multi-index notation

$$
q^{\alpha}(x, y):=q_{1}^{\alpha_{1}}(x, y) \cdots q_{l}^{\alpha_{l}}(x, y) .
$$

Analogously, the notion of an $\mathrm{L}^{*}$-strongly admissible collection suitable for the conjugate problem is that of a family $\widetilde{q}_{j} \in C^{\infty}(\Omega \times \Omega), j=1, \ldots, l$, satisfying the properties:

- The multiplication by $\widetilde{q}_{j}(\cdot, \cdot)$ is a continuous linear mapping on $C_{\mathrm{L}}^{\infty}(\bar{\Omega} \times \bar{\Omega})$, for all $j=1, \ldots, l$;

- $\widetilde{q}_{j}(x, x)=0$ and $\left.\nabla_{y} \widetilde{q}_{j}(x, y)\right|_{y=x} \neq 0$ for all $j=1, \ldots, l$ and all $x \in \Omega$;

- $\left.\operatorname{rank}\left(\nabla_{y} \widetilde{q}_{1}(x, y), \ldots, \nabla_{y} \widetilde{q}_{l}(x, y)\right)\right|_{y=x}=n$ for all $x \in \Omega$;

- the diagonal in $\Omega \times \Omega$ is the only set when all of $\widetilde{q}_{j}$ 's vanish:

$$
\bigcap_{j=1}^{l}\left\{(x, y) \in \Omega \times \Omega: \widetilde{q}_{j}(x, y)=0\right\}=\{(x, x): x \in \Omega\} .
$$

We also write

$$
\widetilde{q}^{\alpha}(x, y):=\widetilde{q}_{1}^{\alpha_{1}}(x, y) \cdots \widetilde{q}_{l}^{\alpha_{l}}(x, y) .
$$

For an operator $A: C_{\mathrm{L}}^{\infty}(\bar{\Omega}) \rightarrow \mathcal{D}_{\mathrm{L}}^{\prime}(\Omega)$ with Schwartz kernel $K_{A}$, let us define $A_{q^{\alpha}}: C_{\mathrm{L}}^{\infty}(\bar{\Omega}) \rightarrow \mathcal{D}_{\mathrm{L}}^{\prime}(\Omega)$ as an operator with the kernel

$$
q^{\alpha}(x, y) K_{A}(x, y)
$$

We understand this formula in the sense of distributions, i.e.

$$
\left\langle q^{\alpha} K_{A}, \varphi\right\rangle:=\left\langle K_{A}, q^{\alpha} \varphi\right\rangle \quad\left(K_{A} \in \mathcal{D}_{\mathrm{L}}^{\prime}(\Omega \times \Omega), \varphi \in C_{\mathrm{L}^{*}}^{\infty}(\bar{\Omega} \times \bar{\Omega})\right) .
$$

Then, we have

$$
A_{q^{\alpha}} f(x)=\int_{\Omega} q^{\alpha}(x, y) K_{A}(x, y) f(y) d y .
$$

Also analogously, for an operator $B: C_{\mathrm{L}^{*}}^{\infty}(\bar{\Omega}) \rightarrow \mathcal{D}_{\mathrm{L}^{*}}^{\prime}(\Omega)$ with Schwartz kernel $K_{B}$, we define $B_{\widetilde{q}^{\alpha}}: C_{\mathrm{L}^{*}}^{\infty}(\bar{\Omega}) \rightarrow \mathcal{D}_{\mathrm{L}^{*}}^{\prime}(\Omega)$ as an operator with the kernel

$$
\widetilde{q}^{\alpha}(x, y) K_{B}(x, y) \text {. }
$$


We understand this formula in the sense of distributions, i.e.

$$
\left\langle\widetilde{q}^{\alpha} K_{B}, \varphi\right\rangle:=\left\langle K_{B}, \widetilde{q}^{\alpha} \varphi\right\rangle \quad\left(K_{B} \in \mathcal{D}_{\mathrm{L}^{*}}^{\prime}(\Omega \times \Omega), \varphi \in C_{\mathrm{L}}^{\infty}(\bar{\Omega} \times \bar{\Omega})\right) .
$$

Then, we get

$$
B_{\widetilde{q}^{\alpha}} f(x)=\int_{\Omega} \widetilde{q}^{\alpha}(x, y) K_{B}(x, y) f(y) d y
$$

Definition 4.1. Let

$$
A: C_{\mathrm{L}}^{\infty}(\bar{\Omega}) \rightarrow \mathcal{D}_{\mathrm{L}}^{\prime}(\Omega)
$$

be an L-admissible operator with the symbol $a \in \mathcal{D}_{\mathrm{L}}^{\prime}(\Omega) \otimes \mathcal{S}^{\prime}(\mathcal{I})$ and with the Schwartz kernel $K_{A} \in \mathcal{D}_{\mathrm{L}}^{\prime}(\Omega \times \Omega)$. Then we define the difference operator

$$
\Delta_{q}^{\alpha}: \mathcal{D}_{\mathrm{L}}^{\prime}(\Omega) \otimes \mathcal{S}^{\prime}(\mathcal{I}) \rightarrow \mathcal{D}_{\mathrm{L}}^{\prime}(\Omega) \otimes \mathcal{S}^{\prime}(\mathcal{I})
$$

acting on L-symbols by

$$
\begin{aligned}
\Delta_{q}^{\alpha} a(x, \xi) & :=u_{\xi}^{-1}(x) \int_{\Omega} K_{A_{q^{\alpha}}}(x, y) u_{\xi}(y) d y \\
& =u_{\xi}^{-1}(x) \int_{\Omega} q^{\alpha}(x, y) K_{A}(x, y) u_{\xi}(y) d y
\end{aligned}
$$

where $K_{A_{q^{\alpha}}} \in \mathcal{D}_{\mathrm{L}}^{\prime}(\Omega \times \Omega)$ is the Schwartz kernel of the L-admissible operator $A_{q^{\alpha}}$ : $C_{\mathrm{L}}^{\infty}(\bar{\Omega}) \rightarrow \mathcal{D}_{\mathrm{L}}^{\prime}(\Omega)$.

Analogously, for the $\mathrm{L}^{*}$-admissible operator

$$
B: C_{\mathrm{L}^{*}}^{\infty}(\bar{\Omega}) \rightarrow \mathcal{D}_{\mathrm{L}^{*}}^{\prime}(\Omega)
$$

with the symbol $b \in \mathcal{D}_{\mathrm{L}^{*}}^{\prime}(\Omega) \otimes \mathcal{S}^{\prime}(\mathcal{I})$ and with the Schwartz kernel $\widetilde{K}_{B} \in \mathcal{D}_{\mathrm{L}^{*}}^{\prime}(\Omega \times \Omega)$ we define the difference operator

$$
\widetilde{\Delta}_{q}^{\alpha}: \mathcal{D}_{\mathrm{L}^{*}}^{\prime}(\Omega) \otimes \mathcal{S}^{\prime}(\mathcal{I}) \rightarrow \mathcal{D}_{\mathrm{L}^{*}}^{\prime}(\Omega) \otimes \mathcal{S}^{\prime}(\mathcal{I})
$$

acting on $\mathrm{L}^{*}$-symbols by

$$
\begin{aligned}
\widetilde{\Delta}_{q}^{\alpha} b(x, \xi) & :=v_{\xi}^{-1}(x) \int_{\Omega} \widetilde{K}_{B_{q^{\alpha}}}(x, y) v_{\xi}(y) d y \\
& =v_{\xi}^{-1}(x) \int_{\Omega} \widetilde{q}^{\alpha}(x, y) \widetilde{K}_{B}(x, y) v_{\xi}(y) d y,
\end{aligned}
$$

where $K_{B_{q^{\alpha}}} \in \mathcal{D}_{\mathrm{L}^{*}}^{\prime}(\Omega \times \Omega)$ is the Schwartz kernel of the $\mathrm{L}^{*}$-admissible operator $B_{q^{\alpha}}: C_{\mathrm{L}^{*}}^{\infty}(\bar{\Omega}) \rightarrow \mathcal{D}_{\mathrm{L}^{*}}^{\prime}(\Omega)$.

We now record the Taylor expansion formula with respect to a family of $q_{j}$ 's, which follows from expansions of functions $g$ and $q^{\alpha}(e, \cdot)$ by the common Taylor series:

Proposition 4.2. Any smooth function $g \in C^{\infty}(\Omega)$ can be approximated by Taylor polynomial type expansions, i.e. for any $e \in \Omega$, we have

$$
\begin{gathered}
g(x)=\left.\sum_{|\alpha|<N} \frac{1}{\alpha !} D_{x}^{(\alpha)} g(x)\right|_{x=e} q^{\alpha}(e, x)+\sum_{|\alpha|=N} \frac{1}{\alpha !} q^{\alpha}(e, x) g_{N}(x) \\
\left.\sim \sum_{\alpha \geq 0} \frac{1}{\alpha !} D_{x}^{(\alpha)} g(x)\right|_{x=e} q^{\alpha}(e, x)
\end{gathered}
$$


in a neighborhood of $e \in \Omega$, where $g_{N} \in C^{\infty}(\Omega)$ and $\left.D_{x}^{(\alpha)} g(x)\right|_{x=e}$ can be found from the recurrent formulae: $D_{x}^{(0, \cdots, 0)}:=I$ and for $\alpha \in \mathbb{N}_{0}^{l}$,

$$
\left.\partial_{x}^{\beta} g(x)\right|_{x=e}=\left.\left.\sum_{|\alpha| \leq|\beta|} \frac{1}{\alpha !}\left[\partial_{x}^{\beta} q^{\alpha}(e, x)\right]\right|_{x=e} D_{x}^{(\alpha)} g(x)\right|_{x=e}
$$

where $\beta=\left(\beta_{1}, \ldots, \beta_{n}\right)$ and $\partial_{x}^{\beta}=\frac{\partial^{\beta_{1}}}{\partial x_{1}^{\beta_{1}}} \cdots \frac{\partial^{\beta_{n}}}{\partial x_{n}^{\beta_{n}}}$.

Analogously, any function $g \in C^{\infty}(\Omega)$ can be approximated by Taylor polynomial type expansions corresponding to the adjoint problem, i.e. we have

$$
\begin{gathered}
g(x)=\left.\sum_{|\alpha|<N} \frac{1}{\alpha !} \widetilde{D}_{x}^{(\alpha)} g(x)\right|_{x=e} \widetilde{q}^{\alpha}(e, x)+\sum_{|\alpha|=N} \frac{1}{\alpha !} \widetilde{q}^{\alpha}(e, x) g_{N}(x) \\
\left.\sim \sum_{\alpha \geq 0} \frac{1}{\alpha !} \widetilde{D}_{x}^{(\alpha)} g(x)\right|_{x=e} \widetilde{q}^{\alpha}(e, x)
\end{gathered}
$$

in a neighborhood of $e \in \Omega$, where $g_{N} \in C^{\infty}(\Omega)$ and $\left.\widetilde{D}_{x}^{(\alpha)} g(x)\right|_{x=e}$ are found from the recurrent formula: $\widetilde{D}^{(0, \cdots, 0)}:=I$ and for $\alpha \in \mathbb{N}_{0}^{l}$,

$$
\left.\partial_{x}^{\beta} g(x)\right|_{x=e}=\left.\left.\sum_{|\alpha| \leq|\beta|} \frac{1}{\alpha !}\left[\partial_{x}^{k} \widetilde{q}^{\alpha}(e, x)\right]\right|_{x=e} \widetilde{D}_{x}^{(\alpha)} g(x)\right|_{x=e},
$$

where $\beta=\left(\beta_{1}, \ldots, \beta_{n}\right)$, and $\partial^{\beta}$ is defined as in Proposition 4.2.

It can be seen that operators $D^{(\alpha)}$ and $\widetilde{D}^{(\alpha)}$ are differential operators of order $|\alpha|$. We will understand them in distributions sense, i.e. for the L-admissible (L'-admissible) operator $A: C_{\mathrm{L}}^{\infty}(\bar{\Omega}) \rightarrow \mathcal{D}_{\mathrm{L}}^{\prime}(\Omega)\left(B: C_{\mathrm{L}^{*}}^{\infty}(\bar{\Omega}) \rightarrow \mathcal{D}_{\mathrm{L}^{*}}^{\prime}(\Omega)\right)$ define the operator $D^{(\alpha)} A\left(\widetilde{D}^{(\alpha)} B\right)$ as an operator with the Schwartz kernel $D_{x}^{(\alpha)} K_{A}(x, y)$ $\left(\widetilde{D}_{x}^{(\alpha)} K_{B}(x, y)\right)$. Then we can act on L-symbols (L'-symbols) by $D^{(\alpha)}\left(\widetilde{D}^{(\alpha)}\right)$.

\section{Symbolic CAlculus}

Using such difference operators and derivatives $D^{(\alpha)}$ from Proposition 4.2 we can now define classes of symbols.

Definition 5.1 (Symbol class $S_{\rho, \delta}^{m}(\bar{\Omega} \times \mathcal{I})$ ). Let $m \in \mathbb{R}, 0 \leq \delta, \rho \leq 1$. Let

$$
A: C_{\mathrm{L}}^{\infty}(\bar{\Omega}) \rightarrow \mathcal{D}_{\mathrm{L}}^{\prime}(\Omega)
$$

be an L-admissible operator with the symbol $a \in \mathcal{D}_{\mathrm{L}}^{\prime}(\Omega) \otimes \mathcal{S}^{\prime}(\mathcal{I})$ and with the Schwartz kernel $K_{A} \in \mathcal{D}_{\mathrm{L}}^{\prime}(\Omega \times \Omega)$. Then the L-symbol class $S_{\rho, \delta}^{m}(\bar{\Omega} \times \mathcal{I})$ consists of such symbols $a(x, \xi)$ which are smooth in $x$ for all $\xi \in \mathcal{I}$, and which satisfy

$$
\left|\Delta_{q}^{\alpha} D_{x}^{(\beta)} a(x, \xi)\right| \leq C_{a \alpha \beta m}\langle\xi\rangle^{m-\rho|\alpha|+\delta|\beta|}
$$

for all $x \in \bar{\Omega}$, for all $\alpha, \beta \geq 0$, and for all $\xi \in \mathcal{I}$. Here we understand $D_{x}^{(\beta)} a(x, \xi)$ as the symbol of the operator $D_{x}^{(\beta)} A$, where the operators $D_{x}^{(\beta)}$ are defined in Proposition 4.2. We will often denote them simply by $D^{(\beta)}$. 
The class $S_{1,0}^{m}(\bar{\Omega} \times \mathcal{I})$ will be often denoted by writing simply $S^{m}(\bar{\Omega} \times \mathcal{I})$. In (5.1), we assume that the inequality is satisfied for $x \in \Omega$ and it extends to the closure $\bar{\Omega}$. Furthermore, we define

$$
S_{\rho, \delta}^{\infty}(\bar{\Omega} \times \mathcal{I}):=\bigcup_{m \in \mathbb{R}} S_{\rho, \delta}^{m}(\bar{\Omega} \times \mathcal{I})
$$

and

$$
S^{-\infty}(\bar{\Omega} \times \mathcal{I}):=\bigcap_{m \in \mathbb{R}} S^{m}(\bar{\Omega} \times \mathcal{I}) .
$$

When we have two L-strongly admissible collections, expressing one in terms of the other similarly to Proposition 4.2 and arguing similarly to [RTW14], we can convince ourselves that for $\rho>\delta$ the definition of the symbol class does not depend on the choice of an L-strongly admissible collection.

Analogously, we define for the $\mathrm{L}^{*}$-admissible operator

$$
B: C_{\mathrm{L}^{*}}^{\infty}(\bar{\Omega}) \rightarrow \mathcal{D}_{\mathrm{L}^{*}}^{\prime}(\Omega)
$$

with the symbol $b \in \mathcal{D}_{\mathrm{L}^{*}}^{\prime}(\Omega) \otimes \mathcal{S}^{\prime}(\mathcal{I})$ and with the Schwartz kernel $\widetilde{K}_{B} \in \mathcal{D}_{\mathrm{L}^{*}}^{\prime}(\Omega \times \Omega)$ the $\mathrm{L}^{*}$-symbol class $\widetilde{S}_{\rho, \delta}^{m}(\bar{\Omega} \times \mathcal{I})$ as the space of such symbols $b(x, \xi)$ which are smooth in $x$ for all $\xi \in \mathcal{I}$, and which satisfy

$$
\left|\widetilde{\Delta}_{(x)}^{\alpha} \widetilde{D}^{(\beta)} b(x, \xi)\right| \leq C_{a \alpha \beta m}\langle\xi\rangle^{m-\rho|\alpha|+\delta|\beta|}
$$

for all $x \in \bar{\Omega}$, for all $\alpha, \beta \geq 0$, and for all $\xi \in \mathcal{I}$. Here we understand $\widetilde{D}^{(\beta)} b(x, \xi)$ as the symbol of the operator $\widetilde{D}^{(\beta)} B$. Similarly, we can define classes $\widetilde{S}_{\rho, \delta}^{\infty}(\bar{\Omega} \times \mathcal{I})$ and $\widetilde{S}^{-\infty}(\bar{\Omega} \times \mathcal{I})$.

If $a \in S_{\rho, \delta}^{m}(\bar{\Omega} \times \mathcal{I})$, it is convenient to denote by $a(X, D)=\mathrm{Op}_{\mathrm{L}}(a)$ the corresponding L-pseudo-differential operator defined by

$$
\mathrm{Op}_{\mathrm{L}}(a) f(x)=a(X, D) f(x):=\sum_{\xi \in \mathcal{I}} u_{\xi}(x) a(x, \xi) \widehat{f}(\xi) .
$$

The set of operators $\mathrm{Op}_{\mathrm{L}}(a)$ of the form (5.2) with $a \in S_{\rho, \delta}^{m}(\bar{\Omega} \times \mathcal{I})$ will be denoted by $\mathrm{Op}_{\mathrm{L}}\left(S_{\rho, \delta}^{m}(\bar{\Omega} \times \mathcal{I})\right)$, or by $\Psi_{\rho, \delta}^{m}(\bar{\Omega} \times \mathcal{I})$. If an operator $A$ satisfies $A \in \mathrm{Op}_{\mathrm{L}}\left(S_{\rho, \delta}^{m}(\bar{\Omega} \times \mathcal{I})\right)$, we denote its L-symbol by $\sigma_{A}=\sigma_{A}(x, \xi), x \in \bar{\Omega}, \xi \in \mathcal{I}$. Naturally, $\sigma_{a(X, D)}(x, \xi)=$ $a(x, \xi)$.

Analogously, if $a \in \widetilde{S}_{\rho, \delta}^{m}(\bar{\Omega} \times \mathcal{I})$, we denote by $a(X, D)=\mathrm{Op}_{\mathrm{L}^{*}}(a)$ the corresponding $\mathrm{L}^{*}$-pseudo-differential operator defined by

$$
\mathrm{Op}_{\mathrm{L}^{*}}(a) f(x)=a(X, D) f(x):=\sum_{\xi \in \mathcal{I}} v_{\xi}(x) a(x, \xi) \widehat{f}_{*}(\xi) .
$$

The set of operators $\operatorname{Op}_{\mathrm{L}^{*}}(a)$ of the form (5.3) with $a \in \widetilde{S}_{\rho, \delta}^{m}(\bar{\Omega} \times \mathcal{I})$ will be denoted by $\mathrm{Op}_{\mathrm{L}^{*}}\left(\widetilde{S}_{\rho, \delta}^{m}(\bar{\Omega} \times \mathcal{I})\right)$, or by $\widetilde{\Psi}_{\rho, \delta}^{m}(\bar{\Omega} \times \mathcal{I})$.

Remark 5.2. (Topology on $\left.S_{\rho, \delta}^{m}(\bar{\Omega} \times \mathcal{I})\left(\widetilde{S}_{\rho, \delta}^{m}(\bar{\Omega} \times \mathcal{I})\right)\right)$. The set $S_{\rho, \delta}^{m}(\bar{\Omega} \times \mathcal{I})\left(\widetilde{S}_{\rho, \delta}^{m}(\bar{\Omega} \times \mathcal{I})\right)$ of symbols has a natural topology. Let us consider the functions $p_{\alpha \beta}^{l}: S_{\rho, \delta}^{m}(\bar{\Omega} \times \mathcal{I}) \rightarrow \mathbb{R}$ 
$\left(\widetilde{p}_{\alpha \beta}^{l}: \widetilde{S}_{\rho, \delta}^{m}(\bar{\Omega} \times \mathcal{I}) \rightarrow \mathbb{R}\right)$ defined by

$$
\begin{array}{r}
p_{\alpha \beta}^{l}(\sigma):=\sup \left[\frac{\left|\Delta_{(x)}^{\alpha} D^{(\beta)} \sigma(x, \xi)\right|}{\langle\xi\rangle^{l-\rho|\alpha|+\delta|\beta|}}:(x, \xi) \in \bar{\Omega} \times \mathcal{I}\right] \\
\left(\widetilde{p}_{\alpha \beta}^{l}(\sigma):=\sup \left[\frac{\left|\widetilde{\Delta}_{(x)}^{\alpha} \widetilde{D}^{(\beta)} \sigma(x, \xi)\right|}{\langle\xi\rangle^{l-\rho|\alpha|+\delta|\beta|}}:(x, \xi) \in \bar{\Omega} \times \mathcal{I}\right]\right) .
\end{array}
$$

Now $\left\{p_{\alpha \beta}^{l}\right\}\left(\left\{\widetilde{p}_{\alpha \beta}^{l}\right\}\right)$ is a countable family of seminorms, and they define a Fréchet topology on $S_{\rho, \delta}^{m}(\bar{\Omega} \times \mathcal{I})\left(\widetilde{S}_{\rho, \delta}^{m}(\bar{\Omega} \times \mathbb{Z})\right)$. Due to the bijective correspondence of $\operatorname{Op}_{\mathrm{L}}\left(S_{\rho, \delta}^{m}(\bar{\Omega} \times \mathcal{I})\right)$ and $S_{\rho, \delta}^{m}(\bar{\Omega} \times \mathcal{I})\left(\mathrm{Op}_{\mathrm{L}^{*}}\left(\widetilde{S}_{\rho, \delta}^{m}(\bar{\Omega} \times \mathcal{I})\right)\right.$ and $\left.\widetilde{S}_{\rho, \delta}^{m}(\bar{\Omega} \times \mathbb{Z})\right)$, this directly topologises also the set of operators. These spaces are not normable, and the topologies have but a marginal role.

The notion of a symbol can be naturally extended to that of an amplitude.

Definition 5.3 (L-amplitudes). The class $\mathcal{A}_{\rho, \delta}^{m}(\bar{\Omega})$ of L-amplitudes consists of the functions $a(x, y, \xi)$ which are smooth in $x$ and $y$ for all $\xi \in \mathcal{I}$, and $a(x, x, \xi)$ is an L-symbol for some L-admissible operator and which satisfy

$$
\left|\Delta_{(x)}^{\alpha} \Delta_{(y)}^{\alpha^{\prime}} D_{x}^{(\beta)} D_{y}^{(\gamma)} a(x, y, \xi)\right| \leq C_{a \alpha \alpha^{\prime} \beta \gamma m}\langle\xi\rangle^{m-\rho\left(|\alpha|+\left|\alpha^{\prime}\right|\right)+\delta(|\beta|+|\gamma|)}
$$

for all $x, y \in \bar{\Omega}$, for all $\alpha, \alpha^{\prime}, \beta, \gamma \geq 0$, and for all $\xi \in \mathcal{I}$. Such a function $a$ will be also called an L-amplitude of order $m \in \mathbb{R}$ of type $(\rho, \delta)$. Formally we may also define

$$
\left(\mathrm{Op}_{\mathrm{L}}(a) f\right)(x):=\sum_{\xi \in \mathcal{I}} \int_{\Omega} u_{\xi}(x) \overline{v_{\xi}(y)} a(x, y, \xi) f(y) d y
$$

for $f \in C_{\mathrm{L}}^{\infty}(\bar{\Omega})$. Sometimes we may denote $\mathrm{Op}_{\mathrm{L}}(a)$ by $a(X, Y, D)$. We also write $\mathcal{A}^{m}(\bar{\Omega}):=\mathcal{A}_{1,0}^{m}(\bar{\Omega})$ as well as

$$
\mathcal{A}^{-\infty}(\bar{\Omega}):=\bigcap_{m \in \mathbb{R}} \mathcal{A}^{m}(\bar{\Omega}) \text { and } \mathcal{A}_{\rho, \delta}^{\infty}(\bar{\Omega}):=\bigcup_{m \in \mathbb{R}} \mathcal{A}_{\rho, \delta}^{m}(\bar{\Omega}) .
$$

Clearly we can regard the L-symbols as a special class of L-amplitudes, namely the ones independent of the middle argument. Analogously, the class $\widetilde{\mathcal{A}}_{\rho, \delta}^{m}(\bar{\Omega})$ of L*amplitudes consists of the functions $a(x, y, \xi)$ which are smooth in $x$ and $y$ for all $\xi \in \mathcal{I}$, and $a(x, x, \xi)$ is an $\mathrm{L}^{*}$-symbol for some $\mathrm{L}^{*}$-admissible operator and which satisfy

$$
\left|\widetilde{\Delta}_{(x)}^{\alpha} \widetilde{\Delta}_{(y)}^{\alpha^{\prime}} \widetilde{D}_{x}^{(\beta)} \widetilde{D}_{y}^{(\gamma)} a(x, y, \xi)\right| \leq C_{a \alpha \beta \gamma m}\langle\xi\rangle^{m-\rho\left(|\alpha|+\left|\alpha^{\prime}\right|\right)+\delta(|\beta|+|\gamma|)}
$$

for all $x, y \in \bar{\Omega}$, for all $\alpha, \alpha^{\prime}, \beta, \gamma \geq 0$, and for all $\xi \in \mathcal{I}$. Formally we may also write

$$
\left(\mathrm{Op}_{\mathrm{L}^{*}}(a) f\right)(x):=\sum_{\xi \in \mathcal{I}} \int_{\Omega} v_{\xi}(x) \overline{u_{\xi}(y)} a(x, y, \xi) f(y) d y
$$

for $f \in C_{\mathrm{L}^{*}}^{\infty}(\bar{\Omega})$. We also write $\widetilde{\mathcal{A}}^{m}(\bar{\Omega}):=\widetilde{\mathcal{A}}_{1,0}^{m}(\bar{\Omega})$ as well as $\widetilde{\mathcal{A}}^{-\infty}(\bar{\Omega}):=\bigcap_{m \in \mathbb{R}} \widetilde{\mathcal{A}}^{m}(\bar{\Omega})$ and $\widetilde{\mathcal{A}}_{\rho, \delta}^{\infty}(\bar{\Omega}):=\bigcup_{m \in \mathbb{R}} \widetilde{\mathcal{A}}_{\rho, \delta}^{m}(\bar{\Omega})$. 
Definition 5.4 (Equivalence of amplitudes). We say that amplitudes $a, a^{\prime}$ are $m(\rho, \delta)$ equivalent $(m \in \mathbb{R}), a \stackrel{m, \rho, \delta}{\sim} a^{\prime}$, if $a-a^{\prime} \in \mathcal{A}_{\rho, \delta}^{m}(\bar{\Omega})$; they are asymptotically equivalent, $a \sim a^{\prime}$ (or $a \stackrel{-\infty}{\sim} a^{\prime}$ if we need additional clarity), if $a-a^{\prime} \in \mathcal{A}^{-\infty}(\bar{\Omega})$. For the corresponding operators we also write $\operatorname{Op}(a) \stackrel{m_{,}, \delta}{\sim} \operatorname{Op}\left(a^{\prime}\right)$ and $\operatorname{Op}(a) \sim \operatorname{Op}\left(a^{\prime}\right)$ (or $\mathrm{Op}(a) \stackrel{-\infty}{\sim} \mathrm{Op}\left(a^{\prime}\right)$ if we need additional clarity), respectively. It is obvious that $\stackrel{m, \rho, \delta}{\sim}$ and $\sim$ are equivalence relations.

From the algebraic point of view, we could handle the amplitudes, symbols, and operators modulo the equivalence relation $\sim$, because the L-pseudo-differential operators form a $*$-algebra with $\operatorname{Op}\left(S^{-\infty}(\bar{\Omega} \times \mathcal{I})\right)$ as a subalgebra.

The next theorem is a prelude to asymptotic expansions, which are the main tool in the symbolic analysis of L-pseudo-differential operators.

Theorem 5.5 (Asymptotic sums of symbols). Let $\left(m_{j}\right)_{j=0}^{\infty} \subset \mathbb{R}$ be a sequence such that $m_{j}>m_{j+1}$, and $m_{j} \rightarrow-\infty$ as $j \rightarrow \infty$, and $\sigma_{j} \in S_{\rho, \delta}^{m_{j}}(\bar{\Omega} \times \mathcal{I})$ for all $j \in \mathcal{I}$. Then there exists an L-symbol $\sigma \in S_{\rho, \delta}^{m_{0}}(\bar{\Omega} \times \mathcal{I})$ such that for all $N \in \mathcal{I}$,

$$
\sigma \stackrel{m_{N}, \rho, \delta}{\sim} \sum_{j=0}^{N-1} \sigma_{j} .
$$

We will now look at the formula for the symbol of the adjoint operator. Let $A \in \mathrm{Op}_{\mathrm{L}}\left(S_{\rho, \delta}^{m}(\bar{\Omega} \times \mathcal{I})\right)$. By the definition of the adjoint operator we have

$$
\left(A u_{\xi}, v_{\eta}\right)_{L^{2}}=\left(u_{\xi}, A^{*} v_{\eta}\right)_{L^{2}}
$$

or

$$
\int_{\Omega} A u_{\xi}(x) \overline{v_{\eta}(x)} d x=\int_{\Omega} u_{\xi}(x) \overline{A^{*} v_{\eta}(x)} d x
$$

for $\xi, \eta \in \mathcal{I}$. Plugging in the integral expressions, we get

$$
\begin{aligned}
\int_{\Omega}\left[\int_{\Omega} K_{A}(x, y) u_{\xi}(y) d y\right] \overline{v_{\eta}(x)} d x & =\int_{\Omega} u_{\xi}(x) \overline{\left[\int_{\Omega} K_{A^{*}}(x, y) v_{\eta}(y) d y\right]} d x \\
& =\int_{\Omega} u_{\xi}(y)\left[\int_{\Omega} \overline{K_{A^{*}}(y, x) v_{\eta}(x)} d x\right] d y
\end{aligned}
$$

for $\xi, \eta \in \mathcal{I}$, where we swapped $x$ and $y$ in the last formula. Consequently, we get the familiar property

$$
K_{A^{*}}(x, y)=\overline{K_{A}(y, x)} .
$$

Now, using this and the equation (3.1), and formula (ii) in Corollary 3.6, and then formula (ii) in Corollary 3.11 and the Taylor expansion in Proposition 4.2, we can write for the $\mathrm{L}^{*}$-symbol $\tau_{A^{*}}$ of $A^{*}$ that

$$
\begin{aligned}
\tau_{A^{*}}(x, \xi) & =v_{\xi}^{-1}(x) \int_{\Omega} K_{A^{*}}(x, y) v_{\xi}(y) d y \\
& =v_{\xi}^{-1}(x) \int_{\Omega} \overline{K_{A}(y, x)} v_{\xi}(y) d y
\end{aligned}
$$




$$
\begin{aligned}
& =v_{\xi}^{-1}(x) \int_{\Omega} \sum_{\eta \in \mathcal{I}} \overline{u_{\eta}(y) \sigma_{A}(y, \eta)} v_{\eta}(x) v_{\xi}(y) d y \\
& \sim v_{\xi}^{-1}(x) \int_{\Omega} \sum_{\eta \in \mathcal{I}} \overline{u_{\eta}(y)} \sum_{\alpha} \frac{1}{\alpha !} \overline{D_{x}^{(\alpha)} \sigma_{A}(x, \eta) q^{\alpha}(x, y)} v_{\eta}(x) v_{\xi}(y) d y
\end{aligned}
$$

as an asymptotic sum. Formally regrouping terms for each $\alpha$, we obtain

$$
\tau_{A^{*}}(x, \xi) \sim \sum_{\alpha} \frac{1}{\alpha !} v_{\xi}^{-1}(x) \int_{\Omega} \sum_{\eta \in \mathcal{I}} \overline{u_{\eta}(y) q^{\alpha}(x, y) D_{x}^{(\alpha)} \sigma_{A}(x, \eta)} v_{\eta}(x) v_{\xi}(y) d y .
$$

Using the formula (3.4), and taking

$$
\widetilde{q}(x, y):=\overline{q(x, y)}
$$

we can write this as

$$
\tau_{A^{*}}(x, \xi) \sim \sum_{\alpha} \frac{1}{\alpha !} \widetilde{\Delta}_{\widetilde{q}}^{\alpha} D_{x}^{(\alpha)} \overline{\sigma_{A}(x, \xi)} .
$$

Making rigorous estimates for the remainder in a routine way, and assuming in the following theorem that for every $x \in \Omega$, the multiplication by $q_{j}(x, \cdot)$ preserves both spaces $C_{\mathrm{L}}^{\infty}(\bar{\Omega})$ and $C_{\mathrm{L}^{*}}^{\infty}(\bar{\Omega})$, we obtained:

Theorem 5.6 (Adjoint operators). Let $0 \leq \delta<\rho \leq 1$. Let $A \in \mathrm{Op}_{\mathrm{L}}\left(S_{\rho, \delta}^{m}(\bar{\Omega} \times \mathcal{I})\right)$. Assume that the conjugate symbol class $\widetilde{S}_{\rho, \delta}^{m}(\bar{\Omega} \times \mathcal{I})$ is defined with strongly admissible functions $\widetilde{q}_{j}(x, y):=\overline{q_{j}(x, y)}$ which are $\mathrm{L}^{*}$-strongly admissible. Then the adjoint of A satisfies $A^{*} \in \mathrm{Op}_{\mathrm{L}^{*}}\left(\widetilde{S}_{\rho, \delta}^{m}(\bar{\Omega} \times \mathcal{I})\right)$, with its $\mathrm{L}^{*}$-symbol $\tau_{A^{*}} \in \widetilde{S}_{\rho, \delta}^{m}(\bar{\Omega} \times \mathcal{I})$ having the asymptotic expansion

$$
\tau_{A^{*}}(x, \xi) \sim \sum_{\alpha} \frac{1}{\alpha !} \widetilde{\Delta}_{x}^{\alpha} D_{x}^{(\alpha)} \overline{\sigma_{A}(x, \xi)} .
$$

We now treat symbols of the amplitude operators.

Theorem 5.7 (Amplitude symbols). Let $0 \leq \delta<\rho \leq 1$ and let $a \in \mathcal{A}_{\rho, \delta}^{m}(\bar{\Omega})$ be such that $\mathrm{Op}_{\mathrm{L}}(a)$ is L-admissible. Then there exists a unique L-symbol $\sigma \in S_{\rho, \delta}^{m}(\bar{\Omega} \times \mathcal{I})$ satisfying $\mathrm{Op}_{\mathrm{L}}(a)=\mathrm{Op}_{\mathrm{L}}(\sigma)$, where

$$
\left.\sigma(x, \xi) \sim \sum_{\alpha \geq 0} \frac{1}{\alpha !} \Delta_{(x)}^{\alpha} D_{y}^{(\alpha)} a(x, y, \xi)\right|_{y=x}
$$

Proof. As a linear operator on $C_{\mathrm{L}}^{\infty}(\bar{\Omega})$, the operator $\mathrm{Op}_{\mathrm{L}}(a)$ possesses the unique Lsymbol $\sigma=\sigma_{\mathrm{Op}_{\mathrm{L}}(a)}$, but at the moment we do not yet know whether $\sigma \in S_{\rho, \delta}^{m}(\bar{\Omega} \times \mathcal{I})$. By Theorem 3.5 the L-symbol is computed from

$$
\sigma(x, \xi)=u_{\xi}^{-1}(x)\left(\mathrm{Op}_{\mathrm{L}}(a) u_{\xi}\right)(x)=u_{\xi}^{-1}(x) \sum_{\eta \in \mathcal{I}} \int_{\Omega} u_{\eta}(x) \overline{v_{\eta}(y)} a(x, y, \eta) u_{\xi}(y) d y .
$$

Now we approximate the function $a(x, \cdot, \eta) \in C^{\infty}(\Omega)$ by Taylor polynomial type expansions, by using Proposition 4.2, we have

$$
\sigma(x, \xi) \sim u_{\xi}^{-1}(x) \sum_{\alpha \geq 0} \frac{1}{\alpha !} \sum_{\eta \in \mathcal{I}} \int_{\Omega} u_{\eta}(x) \overline{v_{\eta}(y)} q^{\alpha}(x, y)\left[D_{y}^{(\alpha)} a(x, y, \eta)\right]_{y=x} u_{\xi}(y) d y
$$




$$
\left.\sim \sum_{\alpha \geq 0} \frac{1}{\alpha !} \Delta_{(x)}^{\alpha} D_{y}^{(\alpha)} a(x, y, \xi)\right|_{y=x}
$$

Omitting a routine verification of the properties of the remainder, this yields the statement.

We now formulate the composition formula.

Theorem 5.8. Let $m_{1}, m_{2} \in \mathbb{R}$ and $\rho>\delta \geq 0$. Let $A, B: C_{\mathrm{L}}^{\infty}(\bar{\Omega}) \rightarrow C_{\mathrm{L}}^{\infty}(\bar{\Omega})$ be linear continuous and $L$-admissible operators, and assume that their L-symbols satisfy

$$
\begin{aligned}
& \left|\Delta_{(x)}^{\alpha} \sigma_{A}(x, \xi)\right| \leq C_{\alpha}\langle\xi\rangle^{m_{1}-\rho|\alpha|}, \\
& \left|D^{(\beta)} \sigma_{B}(x, \xi)\right| \leq C_{\beta}\langle\xi\rangle^{m_{2}+\delta|\beta|},
\end{aligned}
$$

for all $\alpha, \beta \geq 0$, uniformly in $x \in \bar{\Omega}$ and $\xi \in \mathcal{I}$. Then

$$
\sigma_{A B}(x, \xi) \sim \sum_{\alpha \geq 0} \frac{1}{\alpha !}\left(\Delta_{(x)}^{\alpha} \sigma_{A}(x, \xi)\right) D^{(\alpha)} \sigma_{B}(x, \xi),
$$

where the asymptotic expansion means that for every $N \in \mathbb{N}$ we have

$$
\left|\sigma_{A B}(x, \xi)-\sum_{|\alpha|<N} \frac{1}{\alpha !}\left(\Delta_{(x)}^{\alpha} \sigma_{A}(x, \xi)\right) D^{(\alpha)} \sigma_{B}(x, \xi)\right| \leq C_{N}\langle\xi\rangle^{m_{1}+m_{2}-(\rho-\delta) N} .
$$

Proof. First, by the Schwartz kernel theorem from Subsection 2.5, we have

$$
\begin{aligned}
A B f(x) & =\int_{\Omega} K_{A}(x, y)(B f)(y) d y \\
& =\int_{\Omega} K_{A}(x, y)\left[\int_{\Omega} K_{B}(y, z) f(z) d z\right] d y \\
& =\int_{\Omega} \int_{\Omega} K_{A}(x, y) K_{B}(y, z) f(z) d z d y .
\end{aligned}
$$

Hence

$$
\begin{aligned}
\sigma_{A B}(x, \xi) & =u_{\xi}^{-1}(x)\left(A\left(B u_{\xi}\right)\right)(x) \\
& =u_{\xi}^{-1}(x) \int_{\Omega} K_{A}(x, y)\left[\int_{\Omega} K_{B}(y, z) u_{\xi}(z) d z\right] d y \\
& =u_{\xi}^{-1}(x) \int_{\Omega} K_{A}(x, y) u_{\xi}(y) \sigma_{B}(y, \xi) d y .
\end{aligned}
$$

Now we approximate the function $\sigma_{B}(\cdot, \xi) \in C_{\mathrm{L}}^{\infty}(\bar{\Omega})$ by Taylor polynomial type expansions. By using Proposition 4.2, we get

$$
\begin{aligned}
\sigma_{A B}(x, \xi) & \sim u_{\xi}^{-1}(x) \int_{\Omega} K_{A}(x, y)\left[\sum_{\alpha \geq 0} \frac{1}{\alpha !} q^{\alpha}(x, y) D_{x}^{(\alpha)} \sigma_{B}(x, \xi)\right] u_{\xi}(z) d y \\
& =\sum_{\alpha \geq 0} \frac{1}{\alpha !}\left[u_{\xi}^{-1}(x) \int_{\Omega} q^{\alpha}(x, y) K_{A}(x, y) u_{\xi}(y) d y\right] D_{x}^{(\alpha)} \sigma_{B}(x, \xi)
\end{aligned}
$$

Using Definition 4.1, we have

$$
\sigma_{A B}(x, \xi) \sim \sum_{\alpha \geq 0} \frac{1}{\alpha !}\left(\Delta_{(x)}^{\alpha} \sigma_{A}(x, \xi)\right) D_{x}^{(\alpha)} \sigma_{B}(x, \xi) .
$$


Omitting a routine treatment of the remainder, this completes the proof.

\section{ON FURTHER RESUlTS}

6.1. Properties of integral kernels. We now establish some properties of Schwartz kernels of pseudo-differential operators with symbols in the introduced Hörmandertype classes. In the following Theorem 6.1, let us make the assumption on the growth of $L^{\infty}$-norms of the eigenfunctions $u_{\xi}$. Finding estimates for the norms $\left\|u_{\xi}\right\|_{L^{\infty}}$ in terms of the corresponding eigenvalues of $\mathrm{L}$ is a challenging problem even for selfadjoint operators L, see e.g. Sogge and Zelditch [SZ02] and references therein. Thus, on tori or, more generally, on compact Lie groups, the eigenfunctions of the Laplacian can be chosen to be uniformly bounded. However, even for the Laplacian, on more general manifolds, such growth depends on the geometry of the manifold. We refer to [DR14a, Remark 8.9] for a more thorough discussion of this topic as well as for a list of relevant references.

Theorem 6.1 (Kernel of a pseudo-differential operator). Let $\mu_{0}$ be a constant such that there is $C>0$ such that for all $\xi \in \mathcal{I}$ we have

$$
\left\|u_{\xi}\right\|_{L^{\infty}} \leq C\langle\xi\rangle^{\mu_{0}} .
$$

Let $a \in S_{\rho, \delta}^{\mu}(\bar{\Omega} \times \mathcal{I}), \rho>0$. Then the kernel $K(x, y)$ of the pseudo-differential operator $\mathrm{Op}_{\mathrm{L}}$ a satisfies

$$
\left(\mathrm{L}_{y}^{*}\right)^{k}\left(q^{\alpha}(x, y) K(x, y)\right) \in L^{\infty},
$$

for all $|\alpha|>\left(\mu+m k+2 \mu_{0}+s_{0}\right) / \rho$ and $x \neq y$, where $m$ is the order from (1.6) and $s_{0}$ is the constant from Assumption 2.19. If $\mathrm{L}$ is a differential operator it follows that

$$
\left|\left(\mathrm{L}_{y}^{*}\right)^{k} K(x, y)\right| \leq C_{N k}|x-y|^{-N}
$$

for any $N>\left(\mu+m k+2 \mu_{0}+s_{0}\right) / \rho$ and $x \neq y$.

Proof. By Corollary 3.6 we have

$$
u_{\xi}(x) a(x, \xi)=\int_{\Omega} K(x, y) u_{\xi}(y) d y,
$$

and from Definition 4.1 we get

$$
u_{\xi}(x) \Delta_{(x)}^{\alpha} a(x, \xi)=\int_{\Omega} q^{\alpha}(x, y) K(x, y) u_{\xi}(y) d y,
$$

and also

$$
\begin{aligned}
u_{\xi}(x) \lambda_{\xi}^{k} \Delta_{(x)}^{\alpha} a(x, \xi) & =\int_{\Omega} q^{\alpha}(x, y) K(x, y) \lambda_{\xi}^{k} u_{\xi}(y) d y \\
& =\int_{\Omega} q^{\alpha}(x, y) K(x, y) \mathrm{L}_{y}^{k} u_{\xi}(y) d y=\int_{\Omega}\left(\mathrm{L}_{y}^{*}\right)^{k}\left(q^{\alpha}(x, y) K(x, y)\right) u_{\xi}(y) d y .
\end{aligned}
$$

This means that

$$
\left(\mathrm{L}_{y}^{*}\right)^{k}\left(q^{\alpha}(x, y) K(x, y)\right)=\mathcal{F}_{\mathrm{L}}^{-1}\left(u_{\xi}(x) \lambda_{\xi}^{k} \Delta_{(x)}^{\alpha} a(x, \xi)\right)(y) .
$$

Since it follows from assumptions that

$$
\lambda_{\xi}^{k} \Delta_{(x)}^{\alpha} a(x, \xi) \in S^{\mu+m k-\rho|\alpha|}(\bar{\Omega} \times \mathcal{I})
$$


we have

$$
\lambda_{\xi}^{k}\left|\Delta_{(x)}^{\alpha} a(x, \xi)\right| \leq C\langle\xi\rangle^{\mu+m k-\rho|\alpha|}
$$

We recall now the norm

$$
\|a(x, \cdot)\|_{l^{1}(\mathrm{~L})}=\sum_{\xi \in \mathcal{I}}|a(x, \xi)|\left\|u_{\xi}\right\|_{L^{\infty}(\Omega)}
$$

from Subsection 2.4. It follows that

$$
\left\|u_{\xi}(x) \lambda_{\xi}^{k} \Delta_{(x)}^{\alpha} a(x, \xi)\right\|_{l^{1}(\mathrm{~L})} \leq C \sum_{\xi \in \mathcal{I}}\langle\xi\rangle^{\mu+m k-\rho|\alpha|}\left\|u_{\xi}\right\|_{L^{\infty}(\Omega)}^{2} \leq C \sum_{\xi \in \mathcal{I}}\langle\xi\rangle^{\mu+m k-\rho|\alpha|+2 \mu_{0}} .
$$

Consequently, if

$$
|\alpha|>\left(\mu+m k+2 \mu_{0}+s_{0}\right) / \rho
$$

where $s_{0}$ is the constant from Assumption 2.19, we have that $u_{\xi}(x) \lambda_{\xi}^{k} \Delta_{(x)}^{\alpha} a(x, \xi)$ is in $l^{1}(\mathrm{~L})$ with respect to $\xi$, and hence $\left(\mathrm{L}_{y}^{*}\right)^{k}\left(q^{\alpha}(x, y) K(x, y)\right)$ is in $L^{\infty}$ by the HausdorffYoung inequality in Theorem 2.17. Since $\mathrm{L}_{y}^{*}$ is a differential operator for differential operators L, in this case we also have

$$
q^{\alpha}(x, y)\left(\mathrm{L}_{y}^{*}\right)^{k} K(x, y) \in L^{\infty}(\Omega \times \Omega)
$$

for such $\alpha$. By the properties of $q^{\alpha}$ it implies the statement of the theorem.

In particular, if $\mathrm{L}$ is for example locally elliptic, (6.2) implies that for $x \neq y$, the kernel $K(x, y)$ is a smooth function. And, if $a \in S^{-\infty}(\bar{\Omega} \times \mathcal{I})$, then the integral kernel $K(x, y)$ of $\mathrm{Op}_{\mathrm{L}} a$ is smooth in $x$ and $y$.

The singular support of $w \in \mathcal{D}_{\mathrm{L}}^{\prime}(\Omega)$ is defined as the complement of the set where $w$ coincides with a test function. Namely, $x \notin \operatorname{sing} \operatorname{supp} w$ if there is an open neighbourhood $U$ of $x$ and a smooth function $f \in C_{\mathrm{L}}^{\infty}(\bar{\Omega})$ such that $w(\varphi)=f(\varphi)$ for all $\varphi \in C_{\mathrm{L}}^{\infty}(\bar{\Omega})$ with $\operatorname{supp} \varphi \subset U$. As an immediate consequence of Theorem 6.1 we obtain the information on how the singular support is mapped by a pseudo-differential operator:

Corollary 6.2. Let $\sigma_{A} \in S_{\rho, \delta}^{\mu}(\bar{\Omega} \times \mathcal{I}), 1 \geq \rho>\delta \geq 0$. Then for every $w \in \mathcal{D}_{\mathrm{L}}^{\prime}(\Omega)$ we have

$$
\text { sing supp } A w \subset \operatorname{sing} \operatorname{supp} w \text {. }
$$

For elliptic operators, in Corollary 6.4 we state also the inverse inclusion.

6.2. L-elliptic pseudo-differential operators. In this subsection we discuss operators that are elliptic in the symbol classes generated by L. For such operators we can obtain parametrix and then also a-priori estimates by the properties of pseudodifferential operators in, for example, Sobolev spaces, once they are established in Section 6.4, see Theorem 6.8. Thus, from the asymptotic expansion for the composition of pseudo-differential operators, we get an expansion for a parametrix of an elliptic operator:

Theorem 6.3 (L-ellipticity). Let $1 \geq \rho>\delta \geq 0$. Let $\sigma_{A} \in S_{\rho, \delta}^{\mu}(\bar{\Omega} \times \mathcal{I})$ be elliptic in the sense that there exist constants $C_{0}>0$ and $N_{0} \in \mathbb{N}$ such that

$$
\left|\sigma_{A}(x, \xi)\right| \geq C_{0}\langle\xi\rangle^{\mu}
$$


for all $(x, \xi) \in \bar{\Omega} \times \mathcal{I}$ for which $\xi \geq N_{0}$; this is equivalent to assuming that there exists $\sigma_{B} \in S_{\rho, \delta}^{-\mu}(\bar{\Omega} \times \mathcal{I})$ such that $I-B A, I-A B$ are in $\mathrm{Op}_{\mathrm{L}} S^{-\infty}$. Let

$$
A \sim \sum_{j=0}^{\infty} A_{j}
$$

with $\sigma_{A_{j}} \in S_{\rho, \delta}^{\mu-(\rho-\delta) j}(\bar{\Omega} \times \mathcal{I})$. Then

$$
B \sim \sum_{k=0}^{\infty} B_{k}
$$

where $B_{k} \in S_{\rho, \delta}^{-\mu-(\rho-\delta) k}(\bar{\Omega} \times \mathcal{I})$ is such that

$$
\sigma_{B_{0}}(x, \xi)=1 / \sigma_{A_{0}}(x, \xi)
$$

for large enough $\xi$, and recursively

$$
\sigma_{B_{N}}(x, \xi)=\frac{-1}{\sigma_{A_{0}}(x, \xi)} \sum_{k=0}^{N-1} \sum_{j=0}^{N-k} \sum_{|\alpha|=N-j-k} \frac{1}{\alpha !}\left[\Delta_{(x)}^{\alpha} \sigma_{A_{j}}(x, \xi)\right] D_{x}^{(\alpha)} \sigma_{B_{k}}(x, \xi) .
$$

Theorem 6.1 applied to the parametrix from in Theorem 6.3, implies the inverse inclusion to the singular supports from Corollary 6.2 for elliptic operators:

Corollary 6.4. Let $1 \geq \rho>\delta \geq 0$ and assume that $\sigma_{A} \in S_{\rho, \delta}^{\mu}(\bar{\Omega} \times \mathcal{I})$ is L-elliptic. Then for every $w \in \mathcal{D}_{\mathrm{L}}^{\prime}(\Omega)$ we have

$$
\text { sing supp } A w=\operatorname{sing} \operatorname{supp} w .
$$

6.3. Sobolev embedding theorem. In this subsection we give an example of a Sobolev embedding theorem for Sobolev spaces $\mathcal{H}_{\mathrm{L}}^{s}$ associated to L, considered in Section 2.3. However, only limited conclusions are possible in the abstract setting when no further specifics about L are available. Now, let $C(\Omega)$ be the Banach space under the norm

$$
\|f\|_{C(\Omega)}:=\sup _{x \in \Omega}|f(x)| .
$$

We recall that we have a differential operator $L$ of order $m$ with smooth coefficients in the open set $\Omega \subset \mathbb{R}^{n}$, and also the operator $\mathrm{L}^{\circ}$ from (1.7).

The following theorem is conditional to the local regularity estimate (6.4). It is satisfied with $\varkappa=1$ if, for example, L is locally elliptic, i.e. elliptic in the classical sense of $\mathbb{R}^{n}$. However, if $\mathrm{L}$ is for example a sum of squares satisfying Hörmander's commutator condition, the number $\varkappa \geq 1$ may depend on the order to which the Hörmander condition is satisfied, see e.g. [GR15] in the context of compact Lie groups.

Theorem 6.5. Let $k$ be an integer such that $k>n / 2$. Let $\varkappa$ be such that the operators $\mathrm{L}$ and $\mathrm{L}^{\circ}$ satisfy the inequality

$$
\left\|\frac{\partial^{\alpha} f}{\partial x^{\alpha}}\right\|_{L^{2}(\Omega)} \leq C\left\|\left(\mathrm{I}+\mathrm{L}^{\circ} \mathrm{L}\right)^{\frac{\varkappa k}{2 m}} f\right\|_{L^{2}(\Omega)}
$$


for all $f \in C^{\infty}(\Omega)$, for all $\alpha \in \mathbb{N}_{0}^{n}$ with $|\alpha| \leq k$. Then we have the continuous embedding

$$
\mathcal{H}_{\mathrm{L}}^{\varkappa k}(\Omega) \hookrightarrow C(\Omega) .
$$

The proof is similar to [RT16] so we omit it.

6.4. Conditions for $L^{2}$-boundedness. In this subsection we will discuss what conditions on the L-symbol $a$ guarantee the $L^{2}$-boundedness of the corresponding pseudodifferential operator $\operatorname{Op}_{\mathrm{L}}(a): C_{\mathrm{L}}^{\infty}(\bar{\Omega}) \rightarrow \mathcal{D}_{\mathrm{L}}^{\prime}(\Omega)$. The proofs of the following results are similar to [RT16] so we omit them.

Theorem 6.6. Let $k$ be an integer $>n / 2$. Let $a: \bar{\Omega} \times \mathcal{I} \rightarrow \mathbb{C}$ be such that

$$
\left|\partial_{x}^{\alpha} a(x, \xi)\right| \leq C \quad \text { for all }(x, \xi) \in \bar{\Omega} \times \mathcal{I},
$$

and all $|\alpha| \leq k$, all $x \in \Omega$ and $\xi \in \mathcal{I}$. Then the operator $\mathrm{Op}_{\mathrm{L}}(a)$ extends to a bounded operator from $L^{2}(\Omega)$ to $L^{2}(\Omega)$.

From a suitable adaption of the composition Theorem 5.8, using that by Proposition 4.2 the operators $\partial_{x}^{\alpha}$ and $D_{x}^{(\alpha)}$ can be expressed in terms of each other as linear combinations with smooth coefficients, we immediately obtain the result in Sobolev spaces:

Corollary 6.7. Let $k$ be an integer $>n / 2$. Let $\mu \in \mathbb{R}$ and let $a: \bar{\Omega} \times \mathbb{Z} \rightarrow \mathbb{C}$ be such that

$$
\left|\partial_{x}^{\alpha} a(x, \xi)\right| \leq C\langle\xi\rangle^{\mu} \quad \text { for all }(x, \xi) \in \bar{\Omega} \times \mathcal{I},
$$

and for all $\alpha$. Then operator $\mathrm{Op}_{\mathrm{L}}(a)$ extends to a bounded operator from $\mathcal{H}_{L}^{s}(\Omega)$ to $\mathcal{H}_{L}^{s-\mu}(\Omega)$, for any $s \in \mathbb{R}$.

By using Theorem 6.3 and Corollary 6.7, we get

Theorem 6.8. Let $A$ be an L-elliptic pseudo-differential operator with L-symbol $\sigma_{A} \in$ $S^{\mu}(\bar{\Omega} \times \mathcal{I}), \mu \in \mathbb{R}$, and let $A u=f$ in $\Omega, u \in \mathcal{H}_{\mathrm{L}}^{\infty}(\Omega)$. Then we have the estimate

$$
\|u\|_{\mathcal{H}_{\mathrm{L}}^{s+\mu}(\Omega)} \leq C_{s N}\left(\|f\|_{\mathcal{H}_{\mathrm{L}}^{s}(\Omega)}+\|u\|_{\mathcal{H}_{\mathrm{L}}^{-N}(\Omega)}\right) \text {. }
$$

for any $s, N \in \mathbb{R}$.

\section{REFERENCES}

[Bar51] N. K. Bari. Biorthogonal systems and bases in Hilbert space. Moskov. Gos. Univ. Učenye Zapiski Matematika, 148(4):69-107, 1951.

[BL76] J. Bergh and J. Löfström. Interpolation spaces. An introduction. Springer-Verlag, Berlin, 1976. Grundlehren der Mathematischen Wissenschaften, No. 223.

[DR14a] J. Delgado and M. Ruzhansky. Fourier multipliers, symbols and nuclearity on compact manifolds. arXiv:1404.6479, to appear in J. Anal. Math.

[DRT15] J. Delgado, M. Ruzhansky, and N. Tokmagambetov. Schatten classes, nuclearity and nonharmonic analysis on compact manifolds with boundary. arXiv:1505.02261, to appear in J. Math. Pures Appl.

[GR15] C. Garetto and M. Ruzhansky. Wave equation for sums of squares on compact Lie groups. J. Differential Equations, 258(12):4324-4347, 2015.

[KT14] B. Kanguzhin and N. Tokmagambetov. The Fourier transform and convolutions generated by a differential operator with boundary condition on a segment. In Fourier Analysis: Trends in Mathematics, pages 235-251. Birkhäuser Basel AG, Basel, 2014. 
[KTT15] B. Kanguzhin, N. Tokmagambetov, and K. Tulenov. Pseudo-differential operators generated by a non-local boundary value problem. Complex Var. Elliptic Equ., 60(1):107-117, 2015.

[RT16] M. Ruzhansky and N. Tokmagambetov. Nonharmonic analysis of boundary value problems. Int. Math. Res. Not. IMRN, 2016 (12), 3548-3615, 2016.

[RT07] M. Ruzhansky and V. Turunen. On the Fourier analysis of operators on the torus. In Modern trends in pseudo-differential operators, volume 172 of Oper. Theory Adv. Appl., pages 87-105. Birkhäuser, Basel, 2007.

[RT09] M. Ruzhansky and V. Turunen. On the toroidal quantization of periodic pseudodifferential operators. Numer. Funct. Anal. Optim., 30(9-10):1098-1124, 2009.

[RT10a] M. Ruzhansky and V. Turunen. Pseudo-differential operators and symmetries. Background analysis and advanced topics, volume 2 of Pseudo-Differential Operators. Theory and Applications. Birkhäuser Verlag, Basel, 2010.

[RT10b] M. Ruzhansky and V. Turunen. Quantization of pseudo-differential operators on the torus. J. Fourier Anal. Appl., 16(6):943-982, 2010.

[RTW14] M. Ruzhansky, V. Turunen, and J. Wirth. Hörmander class of pseudo-differential operators on compact Lie groups and global hypoellipticity. J. Fourier Anal. Appl., 20(3):476499, 2014.

[SZ02] C. D. Sogge and S. Zelditch. Riemannian manifolds with maximal eigenfunction growth. Duke Math. J., 114(3):387-437, 2002.

Michael Ruzhansky:

Department of Mathematics

IMPERIAL COLLEGE LONDON

180 QueEn's Gate, London, SW7 2AZ

United Kingdom

E-mail address m.ruzhansky@imperial.ac.uk

Niyaz Tokmagambetov:

aL-Farabi KaZakh National University

71 al-Farabi aVe., Almaty, 050040

KAZAKHSTAN,

AND

Department of Mathematics

Imperial College LONDON

180 QueEn's Gate, London, SW7 2AZ

United Kingdom

E-mail address n.tokmagambetov@imperial.ac.uk 